\title{
On a new non-local formulation of water waves
}

\author{
By M. J. ABLOWITZ1, A. S. FOKAS ${ }^{2}$ AND Z. H. MUSSLIMANI \\ ${ }^{1}$ Department of Applied Mathematics, University of Colorado at Boulder, 526 UCB, Boulder, \\ CO 80309-0526, USA \\ ${ }^{2}$ Department of Applied Mathematics and Theoretical Physics, University of Cambridge, \\ Cambridge CB3 0WA, UK \\ ${ }^{3}$ Department of Mathematics, Florida State University, Tallahassee, FL 32306-4510, USA
}

(Received 2 May 2005 and in revised form 3 February 2006)

The classical equations of water waves are reformulated as a system of two equations, one of which is an explicit non-local equation, for the wave height and for the velocity potential evaluated on the free surface. Evaluation of the velocity potential as a function of the depth is not required in order to calculate the wave height and the velocity potential on the free surface. The non-local system yields integral relations related to mass and centre of mass, and is shown to reduce to known asymptotic limits in shallow and deep water. Included in these asymptotic reductions are the Boussinesq, Benney-Luke and nonlinear Schrödinger equations. Two-dimensional lumps with sufficient surface tension are obtained numerically. The extension of this non-local formulation to the case of a variable bottom is also presented.

\section{Introduction}

The study of surface water waves is of wide interest in physics and mathematics. There have been numerous important developments that date back to the time of Stokes and his contemporaries in the nineteenth century. The literature is extensive and in addition to research papers and memoirs there are many books which deal with this subject.

One of the major difficulties in the study of water waves is the determination of the free surface, which appears as an unknown in the basic formulation of the problem. For two dimensional water waves, where the free surface evolves as a function of one space dimension and time, there are various techniques which can be used to eliminate the vertical coordinate and to reduce the problem to the evaluation of the motion of the wave height and velocity potential on the free surface. Effective methods used in the two dimensional water wave problem include conformal mapping and singular integral equations which make use of complex analytic techniques (see e.g. LonguetHiggins \& Cokelet 1976; Fornberg 1980; Dold 1992; Zakharov, Dyachenko \& Vasilyevl 2002). For the three dimensional problem, where the free surface evolves as a function of two space dimensions and time, the situation is more difficult and one loses the possibility of employing complex analysis.

Zakharov (1968) showed that the wave height $\eta$ and velocity potential $\phi$ evaluated on the free surface are canonically conjugate variables and formulated the water wave equations as a Hamiltonian system. Craig \& Sulem (1993) employed these variables and introduced an elegant Dirichlet-Neumann operator $G(\eta)$ associated with the velocity potential, which eliminated the vertical coordinate from the formulation. 
The operator $G(\eta)$ is obtained as a series, which is valid for small $\eta$. This formulation was used in Craig \& Groves (1994) to find small-amplitude/long-wave approximations including the Boussinesq, Korteweg-deVries (KdV) and KadomtsevPetviashvili (KP) equations. In Craig \& Nicholls (2000) this formulation was used to prove the existence of travelling periodic water waves. The DirichletNeumann operator methodology, which employs high-order series approximations to a modified version of $G$, was also employed in Bateman, Swan \& Taylor (2001) and interesting computational investigations were carried out. A collection of some recent mathematical developments in water waves can be found in Craig et al. (2001).

One of the authors has recently introduced a general approach for studying boundary value problems for linear and for integrable nonlinear PDEs (see Fokas 2000). A crucial role in this approach is played by a non-local equation, called the global relation in Fokas (2000), as well as by the equations obtained from the global relation using certain invariant transformations. The analysis of these equations yields an explicit Dirichlet to Neumann map. The employment of the relevant global relation (equation (1.10)) as well as of the proper invariant transformations $(k \rightarrow-k)$, yields the basic non-local equation formulated in this paper.

In this study a new explicit non-local formulation of water waves for both $1+1$ and $2+1$ dimensions is presented. The original equations with unknown boundary conditions are replaced by an integro-differential equation and a nonlinear partial differential equation, both of which are formulated in a known domain. The vertical coordinate is removed from the determining equations. These two equations can be used to determine the wave height and the velocity potential on the free surface. From this system well-known asymptotic equations, in both shallow and deep water with surface tension included, are obtained and agree in the shallow water limit with the results in Craig \& Groves (1994). Furthermore, computational techniques are developed which provide a framework for performing fully nonlinear water wave simulations.

The non-local equation satisfying the Laplace equation, the kinematic and the bottom boundary conditions is found to satisfy the following integral equation in $2+1$ dimensions (see $\S 1.1)$ :

$$
\int_{-\infty}^{\infty} \int_{-\infty}^{\infty} \mathrm{d} x_{1} \mathrm{~d} x_{2} \mathrm{e}^{\mathrm{i} k_{1} x_{1}+\mathrm{i} k_{2} x_{2}}\left\{\mathrm{i} \eta_{t} \cosh [k(\eta+h)]+(\boldsymbol{k} \cdot \nabla q) \frac{\sinh [k(\eta+h)]}{k}\right\}=0,
$$

where $\eta=\eta\left(x_{1}, x_{2}, t\right)$ is the wave height, $q=\phi\left(x_{1}, x_{2}, \eta, t\right)$ is the velocity potential on the surface, $h$ is the unperturbed fluid depth, $k=\sqrt{k_{1}^{2}+k_{2}^{2}}$ and $\boldsymbol{k}=\left(k_{1}, k_{2}\right)$ are 'Fourier-like' parameters.

In $1+1$ dimensions the non-local equation (I) reduces to the convenient form

$$
\int_{-\infty}^{\infty} \mathrm{d} x \mathrm{e}^{\mathrm{i} k x}\left\{\mathrm{i} \eta_{t} \cosh k[\eta+h]+q_{x} \sinh k[\eta+h]\right\}=0, \quad k \text { real. }
$$

Since equations (I), ( $\left.\mathrm{I}^{\prime}\right)$ are valid for all $k_{1}, k_{2}$ real and $k$ real respectively, these equations provide an explicit integral equation formulation of the Dirichlet to Neumann map which represent the summation of the series analysed in Craig \& Sulem (1993). We stress the explicit, spectral and relatively simple form of the free surface equation which is helpful in calculations (e.g. see $\$ \S 2,5-7$ ). 
In terms of the variables $\eta$ and $q$, the dynamic boundary condition takes the form (see $\S 1.1)$

$$
q_{t}+\frac{1}{2}|\nabla q|^{2}+g \eta-\frac{\left(\eta_{t}+\nabla q \cdot \nabla \eta\right)^{2}}{2\left(1+|\nabla \eta|^{2}\right)}=\frac{\sigma}{\rho} \nabla \cdot\left(\frac{\nabla \eta}{\sqrt{1+|\nabla \eta|^{2}}}\right)
$$

where $g, \sigma, \rho$ denote gravity, surface tension and density respectively. Equations (I) and (II) (or equations (I') and (II)) are the two equations that characterize two plus one (or one plus one) dimensional water waves in this formulation.

Some of the results in this paper are the following:

(i) Extensions of the above free surface equations (I), ( $\left.\mathrm{I}^{\prime}\right)$ to the case of varying depth are obtained. Invariance properties related to mass and centre of mass as well as a number of interesting integral relations, including virial-like formulae, are derived using this formulation.

(ii) Two-plus-one-dimensional Boussinesq type systems and the two-plus-onedimensional Benney-Luke type (Benney \& Luke 1964) equation extended to include surface tension are obtained. The Benney-Luke equation reduces to the KP equation for unidirectional waves. The classical one-plus-one-dimensional equations of Boussinesq (Boussinesq 1877) and of Korteweg-deVries (KdV) (Korteweg \& de Vries 1895) in shallow water are obtained as special cases.

(iii) The one-plus-one-dimensional nonlinear Schrödinger equation (cf. Zakharov 1968), which describes the dynamics of small-amplitude, quasi-monochromatic waves with surface tension in deep water, is deduced. Thus, the non-local formulation includes both shallow and deep water limits.

(iv) In shallow water, the non-local formulation yields higher-order nonlinear approximations which go beyond the Boussinesq/Benney-Luke limit. The successive approximations have increasing polynomial nonlinearity: quadratic, cubic, fourth order, etc.

(v) The general time-dependent non-local formulation is shown to reduce properly in the linearized limit, and allows one to compute directly the surface wave height as well as the velocity potential on the free surface.

(vi) A numerical technique applicable to non-local systems, which is an extension of methods used in nonlinear optics (cf. Ablowitz \& Musslimani 2003, 2005), is introduced in order to calculate localized travelling wave solutions to the KadomtsevPetviashvili, Benney-Luke (see also Berger \& Milewski 2000) and the fully nonlinear non-local two-plus-one-dimensional system. These equations are found to have travelling wave lump-soliton-type solutions, provided that sufficient surface tension is included. Methods to obtain one-plus-one-dimensional solitary waves are discussed in the Appendices. The computational results demonstrate that the non-local system derived here can be effectively employed.

In recent experiments (Falcon, Laroache \& Fauve 2002), one dimensional 'depression' solitons in fluids of small depth with sufficient surface tension have been observed for the first time. It may also be feasible to observe travelling lump solitons, which the above numerical results predict to exist in the two-plus-one-dimensional fully nonlinear water wave system, in fluids which have significant surface tension effects.

\subsection{A new Non-local formulation of water waves in three dimensions}

We begin by considering the classical gravity water wave problem without surface tension. Let us define the domain $D$ by

$$
D=\left\{-\infty<x_{j}<\infty, \quad j=1,2, \quad-h<y<\eta\left(x_{1}, x_{2}, t\right), \quad t>0\right\},
$$


where $h>0$. The water wave equations satisfy the following system for $\phi\left(x_{1}, x_{2}, y, t\right)$ and $\eta\left(x_{1}, x_{2}, t\right)$ :

$$
\begin{gathered}
\Delta \phi=0 \text { in } D, \\
\phi_{y}=0 \text { on } y=-h, \\
\eta_{t}+\phi_{x_{1}} \eta_{x_{1}}+\phi_{x_{2}} \eta_{x_{2}}=\phi_{y} \text { on } y=\eta, \\
\phi_{t}+\frac{1}{2}|\nabla \phi|^{2}+g \eta=\frac{\sigma}{\rho} \nabla \cdot\left(\frac{\nabla \eta}{\sqrt{1+|\nabla \eta|^{2}}}\right) \text { on } y=\eta,
\end{gathered}
$$

where $g$ is acceleration due to gravity, $\sigma$ and $\rho$ denote the constant surface tension and density respectively and $h$ is the constant unperturbed fluid depth. Equation (1.3), the kinematic condition, implies that fluid particles on the free surface remain on the free surface, whereas equation (1.4), the so-called dynamic boundary condition or Bernoulli's equation, implies continuity of pressure across the free surface. Equation (1.4) describes the dynamics of the velocity potential on the free surface. We assume that $\eta$ as well as the derivatives of $\phi$ vanish as $x_{1}^{2}+x_{2}^{2} \rightarrow \infty$.

In what follows, we will reformulate these equations in terms of the two functions $\eta$ and $q$, where $q$ is the value of $\phi$ on the free boundary (see Zakharov (1968), where these variables were introduced and shown to be canonically conjugate coordinates),

$$
q\left(x_{1}, x_{2}, t\right)=\phi\left(x_{1}, x_{2}, \eta\left(x_{1}, x_{2}, t\right), t\right) .
$$

The definition of $q$ as well as equation (1.3) can be used to express the spatial derivatives of $\phi$ on the boundary in terms of $\eta$ and $q$. Indeed, differentiating equation (1.5) with respect to $x_{1}$ and $x_{2}$, we find

$$
\begin{aligned}
& \phi_{x_{1}}+\phi_{y} \eta_{x_{1}}=q_{x_{1}}, \\
& \phi_{x_{2}}+\phi_{y} \eta_{x_{2}}=q_{x_{2}} .
\end{aligned}
$$

Solving equations (1.3) and (1.6) for $\phi_{x_{1}}, \phi_{x_{2}}, \phi_{y}$ in terms of $q$ we find

$$
\begin{gathered}
\phi_{x_{1}}=\frac{\left(1+\eta_{x_{2}}^{2}\right) q_{x_{1}}-\eta_{x_{1}} \eta_{x_{2}} q_{x_{2}}-\eta_{x_{1}} \eta_{t}}{1+|\nabla \eta|^{2}} \\
\phi_{x_{2}}=\frac{\left(1+\eta_{x_{1}}^{2}\right) q_{x_{2}}-\eta_{x_{1}} \eta_{x_{2}} q_{x_{1}}-\eta_{x_{2}} \eta_{t}}{1+|\nabla \eta|^{2}} \\
\phi_{y}=\frac{\eta_{t}+\eta_{x_{1}} q_{x_{1}}+\eta_{x_{2}} q_{x_{2}}}{1+|\nabla \eta|^{2}} .
\end{gathered}
$$

\section{The dynamic boundary condition - Bernoulli's equation}

Equations (1.7) imply

$$
|\nabla \phi|^{2}=\frac{\left(1+\eta_{x_{2}}^{2}\right) q_{x_{1}}^{2}+\left(1+\eta_{x_{1}}^{2}\right) q_{x_{2}}^{2}+\eta_{t}^{2}-2 \eta_{x_{1}} \eta_{x_{2}} q_{x_{1}} q_{x_{2}}}{1+|\nabla \eta|^{2}} .
$$

Substituting $|\nabla \phi|^{2}$ in equation (1.4) and also replacing $\phi_{t}$ by $q_{t}-\eta_{t} \phi_{y}$, where $\phi_{y}$ is given by equation $(1.7 c)$, we find an equation involving $q$ and $\eta$. Simplifying this equation we find

$$
q_{t}+\frac{1}{2}|\nabla q|^{2}+g \eta-\frac{\left(\eta_{t}+\nabla q \cdot \nabla \eta\right)^{2}}{2\left(1+|\nabla \eta|^{2}\right)}=\frac{\sigma}{\rho} \nabla \cdot\left(\frac{\nabla \eta}{\sqrt{1+|\nabla \eta|^{2}}}\right),
$$

which is equation (II) in the introduction. 
The analysis of equations (1.1) - (1.3)

Let both functions $\phi\left(x_{1}, x_{2}, y\right)$ and $\psi\left(x_{1}, x_{2}, y\right)$ satisfy Laplace's equation (1.1). Then

$$
\phi_{y}\left(\psi_{x_{1} x_{1}}+\psi_{x_{2} x_{2}}+\psi_{y y}\right)+\psi_{y}\left(\phi_{x_{1} x_{1}}+\phi_{x_{2} x_{2}}+\phi_{y y}\right)=0 .
$$

This identity can be rewritten in the following form:

$$
\left(\phi_{y} \psi_{x_{1}}+\psi_{y} \phi_{x_{1}}\right)_{x_{1}}+\left(\phi_{y} \psi_{x_{2}}+\psi_{y} \phi_{x_{2}}\right)_{x_{2}}+\left(\phi_{y} \psi_{y}-\phi_{x_{1}} \psi_{x_{1}}-\phi_{x_{2}} \psi_{x_{2}}\right)_{y}=0 .
$$

We note that this identity, in contrast to the classical Green's identity for Laplace's equation, involves only derivatives of $\phi$ and not $\phi$ itself.

We choose for $\psi$ the particular solution

$$
E\left(x_{1}, x_{2}, k_{1}, k_{2}\right)=\mathrm{e}^{\mathrm{i} k_{1} x_{1}+\mathrm{i} k_{2} x_{2}+k y}, \quad k=\left(k_{1}^{2}+k_{2}^{2}\right)^{1 / 2} .
$$

Using this particular solution in equation (1.9), we find the identity

$$
\left(E\left(\mathrm{i} k_{1} \phi_{y}+k \phi_{x_{1}}\right)\right)_{x_{1}}+\left(E\left(\mathrm{i} k_{2} \phi_{y}+k \phi_{x_{2}}\right)\right)_{x_{2}}+\left(E\left(k \phi_{y}-\mathrm{i} k_{1} \phi_{x_{1}}-\mathrm{i} k_{2} \phi_{x_{2}}\right)\right)_{y}=0 .
$$

Using the divergence theorem, this equation implies the global relation

$$
\int_{\partial D} E\left\{\left(\mathrm{i} k_{1} \phi_{y}+k \phi_{x_{1}}\right) N_{1}+\left(\mathrm{i} k_{2} \phi_{y}+k \phi_{x_{2}}\right) N_{2}+\left(k \phi_{y}-\mathrm{i} k_{1} \phi_{x_{1}}-\mathrm{i} k_{2} \phi_{x_{2}}\right) N_{3}\right\} \mathrm{d} S=0,
$$

where $\left(N_{1}, N_{2}, N_{3}\right)^{T}$ is a vector normal to the surface $\partial D$, and $\mathrm{d} S$ is a surface element.

On the bottom:

$$
N_{1}=N_{2}=0, \quad N_{3}=-1, \quad y=-h, \quad \phi_{y}=0,
$$

thus the integrand in (1.10) on the bottom becomes

$$
\mathrm{e}^{\mathrm{i} k_{1} x_{1}+\mathrm{i} k_{2} x_{2}-k h}\left(\mathrm{i} k_{1} \phi_{x_{1}}+\mathrm{i} k_{2} \phi_{x_{2}}\right) \text {. }
$$

On the free surface:

$$
N_{1}=-\eta_{x_{1}}, \quad N_{2}=-\eta_{x_{2}}, \quad N_{3}=1, \quad y=\eta,
$$

thus the integrand in (1.10) on the free surface simplifies to

$$
\mathrm{e}^{\mathrm{i} k_{1} x_{1}+\mathrm{i} k_{2} x_{2}+k \eta}\left[k\left(\phi_{y}-\phi_{x_{1}} \eta_{x_{1}}-\phi_{x_{2}} \eta_{x_{2}}\right)-\mathrm{i} k_{1}\left(\phi_{x_{1}}+\phi_{y} \eta_{x_{1}}\right)-\mathrm{i} k_{2}\left(\phi_{x_{2}}+\phi_{y} \eta_{x_{2}}\right)\right] .
$$

The three terms in parentheses appearing in the above expression equal $\eta_{t}, q_{x_{1}}, q_{x_{2}}$, respectively. Thus, with decaying conditions for $|\nabla \phi|$ on the sides, the global relation (1.10) becomes

$$
\begin{aligned}
\int_{-\infty}^{\infty} \int_{-\infty}^{\infty} \mathrm{d} x_{1} \mathrm{~d} x_{2} \mathrm{e}^{\mathrm{i} k_{1} x_{1}+\mathrm{i} k_{2} x_{2}}\left\{\mathrm{e}^{k \eta}\left[k \eta_{t}-\mathrm{i} k_{1} q_{x_{1}}-\mathrm{i} k_{2} q_{x_{2}}\right]\right. \\
\left.+\mathrm{e}^{-k h}\left[\mathrm{i} k_{1} \phi_{x_{1}}+\mathrm{i} k_{2} \phi_{x_{2}}\right]\right\}\left(x_{1}, x_{2},-h, t\right)=0
\end{aligned}
$$

This equation is valid for both roots $\pm \sqrt{k_{1}^{2}+k_{2}^{2}}$. We can eliminate the terms at $y=-h$ from equation (1.11) by replacing $k$ with $-k$. Multiplying by $\mathrm{e}^{k h}$ and subtracting the two equations with $\pm k$ respectively yields equation (I) given in the introduction.

Equations (I) and (II) or (1.8) are the two basic equations for the case of water waves with surface tension. We note that the vertical coordinate $y$ is removed in this formulation, and the unknown boundary $\eta$ is completely determined by the nonlinear integro-differential equation (I) and the nonlinear PDE (1.8). 
We also note that for the case of periodic boundary conditions for the free surface and velocity field, the limits of integration must be modified from an infinite domain to a finite region in order to reflect the size of the period.

\section{The case of a variable bottom}

Let

$$
y=-\tilde{H}\left(x_{1}, x_{2}\right)=-h-H\left(x_{1}, x_{2}\right),
$$

where $h$ is constant, be the equation describing the bottom. Then the normals on the bottom are given by

$$
N_{1}=-H_{x_{1}}, \quad N_{2}=-H_{x_{2}}, \quad N_{3}=-1 .
$$

We also have on the bottom $\mathrm{d} / \mathrm{d} t(z+\tilde{H})=0$, hence

$$
\left.\left(\phi_{y}+\phi_{x_{1}} H_{x_{1}}+\phi_{x_{2}} H_{x_{2}}\right)\left(x_{1}, x_{2},-h-H, t\right)\right)=0 .
$$

Taking

$$
\Phi\left(x_{1}, x_{2}, t\right)=\phi\left(x_{1}, x_{2},-h-H, t\right),
$$

the global relation (1.10) yields

$$
\begin{array}{r}
\int_{-\infty}^{\infty} \int_{-\infty}^{\infty} \mathrm{d} x_{1} \mathrm{~d} x_{2} \mathrm{e}^{\mathrm{i} k_{1} x_{1}+\mathrm{i} k_{2} x_{2}}\left\{\mathrm{e}^{k(\eta+h)}\left[k \eta_{t}-\mathrm{i} k_{1} q_{x_{1}}-\mathrm{i} k_{2} q_{x_{2}}\right]+\mathrm{e}^{-k H}\left[\mathrm{i} k_{1} \Phi_{x_{1}}+\mathrm{i} k_{2} \Phi_{x_{2}}\right]\right\} \\
\left(x_{1}, x_{2},-h, t\right)=0,
\end{array}
$$

where we have used $\Phi_{x_{j}}=\phi_{x_{j}}-\phi_{y} H_{x_{j}}, j=1,2$, and equation (2.1). Replacing $k$ by $-k$ in equation (2.2) and adding and subtracting the resulting equations yields the following two integro-differential equations:

$$
\begin{aligned}
\int_{-\infty}^{\infty} \int_{-\infty}^{\infty} \mathrm{d} x_{1} \mathrm{~d} x_{2} \mathrm{e}^{\mathrm{i} k_{1} x_{1}+\mathrm{i} k_{2} x_{2}}\left\{\mathrm{i} \eta_{t} \cosh [k(\eta+h)]\right. & +(\boldsymbol{k} \cdot \nabla q) \frac{\sinh [k(\eta+h)]}{k} \\
& \left.+(\boldsymbol{k} \cdot \nabla \Phi) \frac{\sinh [k H]}{k}\right\}=0, \\
\int_{-\infty}^{\infty} \int_{-\infty}^{\infty} \mathrm{d} x_{1} \mathrm{~d} x_{2} \mathrm{e}^{\mathrm{i} k_{1} x_{1}+\mathrm{i} k_{2} x_{2}}\left\{\mathrm{i} \eta_{t} \sinh [k(\eta+h)]\right. & +\frac{(\boldsymbol{k} \cdot \nabla q)}{k} \cosh [k(\eta+h)] \\
& \left.-\frac{(\boldsymbol{k} \cdot \nabla \Phi)}{k} \cosh [k H]\right\}=0 .
\end{aligned}
$$

Equations (2.3)-(2.4) and Bernoulli's equation (1.8) are three equations for the three unknowns $\eta, q, \Phi$.

\section{Integral relations}

An immediate consequence of equations (2.3)-(2.4) is mass conservation. If we take $k_{j} \rightarrow 0, j=1,2$, in equation (2.3) and expand, then we find a sequence of integral relations. The first is conservation of mass for any bottom $H\left(x_{1}, x_{2}\right)$ :

$$
\frac{\partial}{\partial t} \int_{-\infty}^{\infty} \int_{-\infty}^{\infty} \mathrm{d} x_{1} \mathrm{~d} x_{2} \eta\left(x_{1}, x_{2}, t\right)=0 .
$$


Some of the additional integral relationships, obtained in this way, which include centre of mass and virial-type formulae, are listed below:

$$
\begin{gathered}
\frac{\partial}{\partial t} \int_{-\infty}^{\infty} \int_{-\infty}^{\infty} \mathrm{d} x_{1} \mathrm{~d} x_{2}\left(x_{j} \eta\right)=\int_{-\infty}^{\infty} \int_{-\infty}^{\infty} \mathrm{d} x_{1} \mathrm{~d} x_{2}\left(q_{x_{j}}(\eta+h)+H \Phi_{x_{j}}\right) \\
\frac{\partial}{\partial t} \int_{-\infty}^{\infty} \int_{-\infty}^{\infty} \mathrm{d} x_{1} \mathrm{~d} x_{2}\left(\frac{x_{j}^{2} \eta}{2}-\left(\frac{\eta^{3}}{6}+\frac{\eta^{2} h}{2}\right)\right)=\int_{-\infty}^{\infty} \int_{-\infty}^{\infty} \mathrm{d} x_{1} \mathrm{~d} x_{2}\left(x_{j} q_{x_{j}}(\eta+h)+x_{j} H \Phi_{x_{j}}\right) \\
\int_{-\infty}^{\infty} \int_{-\infty}^{\infty} \mathrm{d} x_{1} \mathrm{~d} x_{2}\left(q_{x_{j}}-\Phi_{x_{j}}\right)=0
\end{gathered}
$$

where $j=1,2$. When $H=0$, equations (2.6)-(2.7) simplify considerably since the velocity potential, $\Phi$, on the bottom multiplies $H$. Equation (2.6) is the conservation law associated with the motion of the centre of mass. Also, when $H=0$ the right-hand side of (2.6) can be related to the momentum of the fluid $P_{j}, j=1,2$, where

$$
P_{j}=\int_{-\infty}^{\infty} \int_{-\infty}^{\infty} \mathrm{d} x_{1} \mathrm{~d} x_{2} \int_{-h}^{\eta} \mathrm{d} y\left(\rho \phi_{x_{j}}\right),
$$

and $\phi_{x_{j}}, j=1,2$, correspond to the transverse velocities in the water wave equations (1.1)-(1.4). Since the momentum is a known conserved quantity in water waves, we find that the centre of mass equation can also be written as a conserved quantity

$$
\frac{\partial}{\partial t}\left\{\int_{-\infty}^{\infty} \int_{-\infty}^{\infty} \mathrm{d} x_{1} \mathrm{~d} x_{2}\left(x_{j} \eta\right)-t \int_{-\infty}^{\infty} \int_{-\infty}^{\infty} \mathrm{d} x_{1} \mathrm{~d} x_{2} q_{x_{j}}(\eta+h)\right\}=0,
$$

where $j=1,2$. Thus, the centre of mass increases linearly with time. We note that the relation between the centre of mass and the momentum was found in Benjamin \& Mahony (1971). The point symmetries and conserved quantities associated with water waves were derived and discussed in Benjamin \& Olver (1982).

It is also interesting that the analogue of the centre of mass relation (2.6) can be related asymptotically to a non-trivial conserved quantity for the KadomtsevPetviashvili and Korteweg-deVries equations. This is discussed further in $\S 5$.

Recently, the case of a variable bottom was considered in Craig et al. (2005) using an extension of the Dirichlet-Neumann map technique mentioned earlier (Craig \& Sulem 1993). In this paper the authors find wave equations governing small-amplitude, long waves over a periodically varying bottom. In the case of a variable bottom the series expansion for the Dirichlet-Neumann map presented in Craig et al. (2005) is implicit. The explicit non-local equations (2.3)-(2.4) provide the summation of all terms in the equivalent Dirichlet-Neumann series.

It should be mentioned that there are some differences in the case of infinite depth $h \rightarrow \infty$. In this case equation (I) in $\S 1$ takes the following simplified form:

$$
\int_{-\infty}^{\infty} \int_{-\infty}^{\infty} \mathrm{d} x_{1} \mathrm{~d} x_{2} \mathrm{e}^{\mathrm{i} k_{1} x_{1}+\mathrm{i} k_{2} x_{2}} \mathrm{e}^{k \eta}\left\{\mathrm{i} \eta_{t}+\frac{(\boldsymbol{k} \cdot \nabla q)}{k}\right\}=0 .
$$

In this case the integral relations can be obtained either by taking $h \rightarrow \infty$ in the earlier formulae (2.6)-(2.8) with $\Phi_{x_{j}}=0, j=1,2$, or taking the limit as $k_{j} \rightarrow 0$ in equation (ID). In either way, we find equation (2.5) and the following relations:

$$
\int_{-\infty}^{\infty} \int_{-\infty}^{\infty} \mathrm{d} x_{1} \mathrm{~d} x_{2}\left(q_{x_{j}}\right)=0
$$




$$
\begin{gathered}
\frac{\partial}{\partial t} \int_{-\infty}^{\infty} \int_{-\infty}^{\infty} \mathrm{d} x_{1} \mathrm{~d} x_{2}\left(x_{j} \eta\right)=\int_{-\infty}^{\infty} \int_{-\infty}^{\infty} \mathrm{d} x_{1} \mathrm{~d} x_{2}\left(q_{x_{j}} \eta\right), \\
\frac{\partial}{\partial t} \int_{-\infty}^{\infty} \int_{-\infty}^{\infty} \mathrm{d} x_{1} \mathrm{~d} x_{2}\left(\frac{\eta^{2}}{2}\right)=\int_{-\infty}^{\infty} \int_{-\infty}^{\infty} \mathrm{d} x_{1} \mathrm{~d} x_{2}\left(x_{j} q_{x_{j}}\right), \\
\frac{\partial}{\partial t} \int_{-\infty}^{\infty} \int_{-\infty}^{\infty} \mathrm{d} x_{1} \mathrm{~d} x_{2}\left(\frac{x_{j}^{2} \eta}{2}-\frac{\eta^{3}}{6}\right)=\int_{-\infty}^{\infty} \int_{-\infty}^{\infty} \mathrm{d} x_{1} \mathrm{~d} x_{2}\left(x_{j} q_{x_{j}} \eta\right),
\end{gathered}
$$

where $j=1,2$. It is interesting to note that in infinitely deep water one needs the constraints in (2.9), otherwise rapid decay of the wave amplitude $\eta$ and of $\nabla q$ as $r \rightarrow \infty$ do not follow. A discussion of the infinite depth case can be found in Benjamin \& Olver (1982).

In what follows we shall concentrate on the simpler case of a constant bottom $y=-h$. We note that equation (2.3) reduces to equation (I) when $H=0$.

\section{The linear limit}

In the linear limit, $|\eta| \ll 1,\left|q_{t}\right| \ll 1,\left|q_{x_{j}}\right| \ll 1, j=1,2$, thus equations (I) and (1.8) become

$$
\begin{gathered}
\int_{-\infty}^{\infty} \int_{-\infty}^{\infty} \mathrm{d} x_{1} \mathrm{~d} x_{2} \mathrm{e}^{\mathrm{i} k_{1} x_{1}+\mathrm{i} k_{2} x_{2}}\left\{\mathrm{i} \eta_{t} \cosh [k h]+\left[\left(\frac{k_{1}}{k}\right) q_{x_{1}}+\left(\frac{k_{2}}{k}\right) q_{x_{2}}\right] \sinh [k h]\right\}=0, \\
q_{t}+g \eta=\frac{\sigma}{\rho}\left(\eta_{x_{1} x_{1}}+\eta_{x_{2} x_{2}}\right) .
\end{gathered}
$$

We define the Fourier transforms (FT) of $\eta$ by

$$
\hat{\eta}=\int_{-\infty}^{\infty} \int_{-\infty}^{\infty} \mathrm{d} x_{1} \mathrm{~d} x_{2} \mathrm{e}^{\mathrm{i} k_{1} x_{1}+\mathrm{i} k_{2} x_{2}} \eta\left(x_{1}, x_{2}, t\right),
$$

and similarly for the derivatives of $q$.

Using these definitions, equations (3.1) and (3.2) yield

$$
\begin{gathered}
\mathrm{i} \hat{\eta}_{t}+\left[\left(\frac{k_{1}}{k}\right)\left(\widehat{q_{x_{1}}}\right)+\left(\frac{k_{2}}{k}\right)\left(\widehat{q_{x_{2}}}\right)\right] \tanh [k h]=0, \\
\widehat{q_{t}}+g \hat{\eta}=\frac{\sigma}{\rho}\left[\left(\widehat{\left(\eta_{x_{1} x_{2}}\right.}\right)+\left(\widehat{\eta_{x_{2} x_{2}}}\right)\right] .
\end{gathered}
$$

The definition of the FT implies $\left(\widehat{\eta_{x_{1}}}\right)=-\mathrm{i} k_{1} \hat{\eta},\left(\widehat{\eta_{x_{2}}}\right)=-\mathrm{i} k_{2} \hat{\eta}$, etc. Differentiating equation (3.3) with respect to $t$ and using equation (3.4) to express $\widehat{q_{x_{1}} t}$ and $\widehat{q_{x_{2}} t}$ in terms of $\hat{\eta}$, i.e. using

$$
\widehat{q_{t x_{j}}}=\mathrm{i} k_{j}\left(g+\frac{\sigma}{\rho} k^{2}\right) \hat{\eta}, \quad j=1,2,
$$

we find

$$
\hat{\eta}_{t t}+\left[k g\left(1+\frac{\sigma}{g \rho} k^{2}\right) \tanh [k h]\right] \hat{\eta}=0
$$

\section{The non-dimensional form}

It is convenient to have a non-dimensional formulation. In this respect, we first replace all variables in equations (I) and (1.8) by prime variables and then we make 
the following substitutions:

$$
x_{1}^{\prime}=l x_{1}, \quad x_{2}^{\prime}=\frac{l}{\gamma} x_{2}, \quad k_{1}^{\prime}=\frac{k_{1}}{l}, \quad k_{2}^{\prime}=\gamma \frac{k_{2}}{l}, \quad t^{\prime}=\frac{l t}{c_{0}}, \quad q^{\prime}=\frac{g l a}{c_{0}} q, \quad \eta^{\prime}=a \eta,
$$

where $c_{0}=\sqrt{g h}$ and $l, l / \gamma$ are typical length scales (e.g. wavelengths) in the $x_{1-}$, $x_{2}$-directions respectively. Defining the dimensionless parameters

$$
\varepsilon=\frac{a}{h}, \quad \mu=\frac{h}{l},
$$

equations (I) and (1.8) yield the following equations:

$$
\begin{gathered}
\int_{-\infty}^{\infty} \int_{-\infty}^{\infty} \mathrm{d} x_{1} \mathrm{~d} x_{2} \mathrm{e}^{\mathrm{i}\left(k_{1} x_{1}+k_{2} x_{2}\right)}\left\{\mathrm{i} \eta_{t} \cosh [k \mu(1+\varepsilon \eta)]+\sinh [k \mu(1+\varepsilon \eta)]\right. \\
\left.\times\left[\frac{k_{1}}{k \mu} q_{x_{1}}+\frac{\gamma^{2} k_{2}}{k \mu} q_{x_{2}}\right]\right\}=0, \\
q_{t}+\eta+\frac{\varepsilon}{2}\left(q_{x_{1}}^{2}+\gamma^{2} q_{x_{2}}^{2}\right)-\frac{1}{2} \varepsilon \mu^{2} \frac{\left[\left(\eta_{t}+\varepsilon\left(q_{x_{1}} \eta_{x_{1}}+\gamma^{2} q_{x_{2}} \eta_{x_{2}}\right)\right]^{2}\right.}{1+(\varepsilon \mu)^{2}\left(\eta_{x_{1}}^{2}+\gamma^{2} \eta_{x_{2}}^{2}\right)} \\
=\frac{\tilde{\sigma}\left[\mu^{2} \eta_{x_{1} x_{1}}\left(1+\varepsilon^{2} \mu^{2} \gamma^{2} \eta_{x_{2}}^{2}\right)+\mu^{2} \gamma^{2} \eta_{x_{2} x_{2}}\left(1+\varepsilon^{2} \mu^{2} \eta_{x_{1}}^{2}\right)-2 \varepsilon^{2} \mu^{4} \gamma^{2} \eta_{x_{1}} \eta_{x_{2}} \eta_{x_{1} x_{2}}\right]}{\left[1+(\varepsilon \mu)^{2}\left(\eta_{x_{1}}^{2}+\gamma^{2} \eta_{x_{2}}^{2}\right)\right]^{3 / 2}},
\end{gathered}
$$

where

$$
k=\sqrt{k_{1}^{2}+\gamma^{2} k_{2}^{2}}, \quad \tilde{\sigma}=\frac{\sigma}{\rho g h^{2}} .
$$

By adding/subtracting the linear terms and by taking the Fourier transform, we can rewrite equations (4.1) and (4.2) in an alternative form in which the linear terms are separated. This is useful for various purposes, including for numerical evaluations (see $\S 7$ on computational investigations):

$$
\begin{aligned}
\mathrm{i} \cosh [k \mu] \hat{\eta}_{t} & +\sinh [k \mu]\left[\left(\frac{k_{1}}{k \mu}\right)\left(\widehat{q_{x_{1}}}\right)+\left(\frac{\gamma^{2} k_{2}}{k \mu}\right)\left(\widehat{q_{x_{2}}}\right)\right] \\
= & \int_{-\infty}^{\infty} \int_{-\infty}^{\infty} \mathrm{d} x_{1} \mathrm{~d} x_{2} \mathrm{e}^{\mathrm{i}\left(k_{1} x_{1}+k_{2} x_{2}\right)}\left\{\mathrm{i} \eta_{t}(\cosh [k \mu]-\cosh [k \mu(1+\varepsilon \eta)])\right. \\
& \left.+(\sinh [k \mu]-\sinh [k \mu(1+\varepsilon \eta)])\left[\frac{k_{1}}{k \mu} q_{x_{1}}+\frac{\gamma^{2} k_{2}}{k \mu} q_{x_{2}}\right]\right\} \\
\hat{q}_{t}+\left(1+\tilde{\sigma} \mu^{2} k^{2}\right) \hat{\eta}= & \int_{-\infty}^{\infty} \int_{-\infty}^{\infty} \mathrm{d} x_{1} \mathrm{~d} x_{2} \mathrm{e}^{\mathrm{i}\left(k_{1} x_{1}+k_{2} x_{2}\right)}\left\{-\frac{\varepsilon}{2}\left(q_{x_{1}}^{2}+\gamma^{2} q_{x_{2}}^{2}\right)\right. \\
& +\frac{1}{2} \varepsilon \mu^{2} \delta^{-1}\left[\eta_{t}+\varepsilon\left(q_{x_{1}} \eta_{x_{1}}+\gamma^{2} q_{x_{2}} \eta_{x_{2}}\right)\right]^{2} \\
& +\delta^{-3 / 2} \tilde{\sigma}\left[\mu^{2} \eta_{x_{1} x_{1}}\left(1+\varepsilon^{2} \mu^{2} \gamma^{2} \eta_{x_{2}}^{2}\right)+\mu^{2} \gamma^{2} \eta_{x_{2} x_{2}}\left(1+\varepsilon^{2} \mu^{2} \eta_{x_{1}}^{2}\right)\right. \\
& \left.\left.-2 \varepsilon^{2} \mu^{4} \gamma^{2} \eta_{x_{1}} \eta_{x_{2}} \eta_{x_{1} x_{2}}-\delta^{3 / 2} \mu^{2}\left(\eta_{x_{1} x_{1}}+\gamma^{2} \eta_{x_{2} x_{2}}\right)\right]\right\}
\end{aligned}
$$

where

$$
\delta=1+(\varepsilon \mu)^{2}\left(\eta_{x_{1}}^{2}+\gamma^{2} \eta_{x_{2}}^{2}\right)
$$


Equations (4.4) and (4.2) (or its integral form (4.5)), are a system of two equations for the two unknowns, $\eta$ and $q$. It is also worth noting that equation (4.4) can be written in terms of the following integral equation for the function $\eta_{t}$ :

where

$$
\hat{\eta}_{t}=\int_{-\infty}^{\infty} \int_{-\infty}^{\infty} \mathrm{d} x_{1} \mathrm{~d} x_{2} \mathrm{e}^{\mathrm{i}\left(k_{1} x_{1}+k_{2} x_{2}\right)} K[\eta(t)] \eta_{t}+\mathscr{F}[\eta(t), Q(t)]
$$

and

$$
K(\eta)=\frac{\cosh [k \mu]-\cosh [k \mu(1+\varepsilon \eta)]}{\cosh (k \mu)}
$$

$$
F(\eta, Q)=\int_{-\infty}^{\infty} \int_{-\infty}^{\infty} \mathrm{d} x_{1} \mathrm{~d} x_{2} \mathrm{e}^{\mathrm{i}\left(k_{1} x_{1}+k_{2} x_{2}\right)}\left(\frac{\mathrm{i} \sinh [k \mu(1+\varepsilon \eta)]}{\cosh [k \mu]}\left[\frac{k_{1}}{k \mu} q_{x_{1}}+\frac{\gamma^{2} k_{2}}{k \mu} q_{x_{2}}\right]\right) .
$$

This integral equation provides the summation of the Dirichlet-Neumann series operator discussed in Craig \& Sulem (1993), Craig \& Nicholls (2000) and Bateman et al. (2001). Equations (4.6) and (4.2) (or (4.5)), are the closed system of water wave equations. Equation (4.6) is formulated as an integral equation for $\eta_{t}$. Equation (4.5) determines $\hat{q}_{t}$ as a quadratically nonlinear function of $\eta_{t}$.

\section{Two dimensional Boussinesq, Benney-Luke and KP equations}

In this section, starting from the non-local formulation, we derive the Boussinesq, Benney-Luke and KP equations with surface tension. We include surface tension because later we will address the question of finding lump-type solutions to the water wave equations. We take $|\varepsilon| \ll 1,|\mu| \ll 1$, use the expansions

$$
\cosh [k \mu(1+\varepsilon \eta)] \sim 1+\frac{\mu^{2}}{2} k^{2}, \quad \sinh [k \mu(1+\varepsilon \eta)] \sim \mu k+\frac{\mu^{3}}{6} k^{3}+\varepsilon \mu \eta k,
$$

and we use integration by parts, as well as properties of the Fourier transform

$$
k_{j} \rightarrow \mathrm{i} \partial x_{j}, \quad j=1,2 .
$$

Then after some algebra we find that to within $O\left(\varepsilon, \mu^{2}\right)$, equations (4.1) and (4.2) yield the following Boussinesq-type equations, where for convenience we replace $x_{1}, x_{2}$ by $x, y$ :

$$
\begin{gathered}
\left(1-\frac{\mu^{2}}{2} \tilde{\Delta}\right) \eta_{t}+\left(\tilde{\Delta}-\frac{\mu^{2}}{6} \tilde{\Delta}^{2}\right) q+\varepsilon\left(\eta_{x} q_{x}+\gamma^{2} \eta_{y} q_{y}\right)+\varepsilon \eta \tilde{\Delta} q=0, \\
\eta=-q_{t}-\frac{\varepsilon}{2}\left(q_{x}^{2}+\gamma^{2} q_{y}^{2}\right)+\tilde{\sigma} \mu^{2} \tilde{\Delta} \eta
\end{gathered}
$$

where

$$
\tilde{\Delta}=\partial_{x}^{2}+\gamma^{2} \partial_{y}^{2}
$$

Equation (5.2) implies

$$
\eta \sim-\left(1+\tilde{\sigma} \mu^{2} \tilde{\Delta}\right) q_{t}-\frac{\varepsilon}{2}\left(q_{x}^{2}+\gamma^{2} q_{y}^{2}\right)
$$

Substituting this expression in equation (5.1) we find

$$
\left[1+\left(\tilde{\sigma}-\frac{1}{2}\right) \mu^{2} \tilde{\Delta}\right] q_{t t}-\left(\tilde{\Delta}-\frac{\mu^{2}}{6} \tilde{\Delta}^{2}\right) q+\varepsilon\left(2 q_{x} q_{x t}+2 \gamma^{2} q_{y} q_{y t}+q_{t} \tilde{\Delta} q\right)=0 .
$$


Using the leading-order approximation, $q_{t t} \sim \tilde{\Delta} q$, equation (5.4) implies the following equation, valid to $O\left(\varepsilon, \mu^{2}\right)$ :

$$
q_{t t}-\tilde{\Delta} q+\left(\tilde{\sigma}-\frac{1}{3}\right) \mu^{2} \tilde{\Delta}^{2} q+\varepsilon\left(\partial_{t}|\tilde{\nabla} q|^{2}+q_{t} \tilde{\Delta} q\right)=0,
$$

where $|\tilde{\nabla} q|^{2}=\left(q_{x}^{2}+\gamma^{2} q_{y}^{2}\right)$.

An asymptotically equivalent equation to (5.5) was derived by Benney \& Luke (1964) in terms of the velocity potential $\phi$ in the case of zero surface tension $(\tilde{\sigma}=0)$. We note that $q \sim \phi+O\left(\varepsilon \mu^{2}\right)$, which allows us to establish the relationship between the Benney-Luke equation for $\phi$ and the above equation (5.5) for $q$.

If we take

$$
\gamma=O(\mu), \quad \varepsilon=O\left(\mu^{2}\right)
$$

then further simplifications can be made. In this case, (5.5) becomes

$$
q_{t t}-q_{x x}+\left(\tilde{\sigma}-\frac{1}{3}\right) \mu^{2} q_{x x x x}-\gamma^{2} q_{y y}+\varepsilon\left(2 q_{x} q_{x t}+q_{t} q_{x x}\right)=0 .
$$

Let

$$
\xi=x-t, \quad T=\varepsilon t,
$$

thus

Then, equation (5.5) simplifies to

$$
\partial_{t}=-\partial_{\xi}+\varepsilon \partial_{T}, \quad \partial_{x}=\partial_{\xi} .
$$

$$
2 \varepsilon q_{T \xi}+\left(\frac{1}{3}-\tilde{\sigma}\right) \mu^{2} q_{\xi \xi \xi \xi}+\gamma^{2} q_{y y}+3 \varepsilon q_{\xi} q_{\xi \xi}=0 .
$$

Letting $w=q_{\xi}, \varepsilon=\mu^{2}=\gamma^{2}$, and taking a derivative with respect to $\xi$, this equation becomes

$$
2 w_{T \xi}+\left(\frac{1}{3}-\tilde{\sigma}\right) w_{\xi \xi \xi \xi}+w_{y y}+\left(3 w w_{\xi}\right)_{\xi}=0
$$

which is the well known Kadomtsev-Petviashvili (KP) equation (Kadomtsev \& Petviashvili 1970). For convenience we put the KP equation (5.7) in standard form by making the following transformations:

$$
\left.\begin{array}{l}
w=-2 \operatorname{sgn}\left(\tilde{\sigma}-\frac{1}{3}\right) l_{1}^{2} u, \xi \rightarrow l_{1} x, y \rightarrow \frac{1}{\sqrt{3}} y, \\
T=-\frac{2 \operatorname{sgn}\left(\tilde{\sigma}-\frac{1}{3}\right) \tau}{l_{1}}, \quad l_{1}=\left|\tilde{\sigma}-\frac{1}{3}\right|^{1 / 4} ;
\end{array}\right\}
$$

then $u$ satisfies the standard KP equation

$$
u_{\tau x}+u_{x x x x}-3 \operatorname{sgn}\left(\tilde{\sigma}-\frac{1}{3}\right) u_{y y}+6\left(u u_{x}\right)_{x}=0 .
$$

We note that the KdV equation is a reduction of KP by considering the $y$-independent solutions.

The KP equation is well known to have lump-type soliton solutions (cf. Ablowitz $\&$ Clarkson 1991) for $\left(\tilde{\sigma}-\frac{1}{3}\right)>0$. The 1-lump solution is given by the formula

$$
u=16 \frac{-4\left(x^{\prime}-2 k_{R} y^{\prime}\right)^{2}+16 k_{I}^{2} y^{\prime 2}+1 / k_{I}^{2}}{\left[4\left(x^{\prime}-2 k_{R} y^{\prime}\right)^{2}+16 k_{I}^{2} y^{\prime 2}+1 / k_{I}^{2}\right]^{2}},
$$

where

$$
x^{\prime}=x-c_{x} \tau-x_{0}, \quad y^{\prime}=y-c_{y} \tau-y_{0}, \quad c_{x}=12\left(k_{R}^{2}+k_{I}^{2}\right), \quad c_{y}=12 k_{R} ;
$$

the velocity satisfies the condition

$$
c_{x}>\frac{c_{y}^{2}}{12}
$$


This has implications regarding the existence of a 1-lump solution for the BenneyLuke (BL) equation when $\left(\tilde{\sigma}-\frac{1}{3}\right)>0$ (see also the discussion in the computational section, §7). In terms of the original water wave variables, we have

$$
\begin{gathered}
x^{\prime}=\frac{1}{l_{1}}\left(\xi+\frac{c_{x} l_{1}^{2} T}{2}\right)=\frac{1}{l_{1}}\left[x-\left(1-\frac{l_{1}^{2} \varepsilon c_{x}}{2}\right) t\right], \\
y^{\prime}=\sqrt{3}\left(x_{2}+\frac{c_{y} l_{1} T}{2 \sqrt{3}}\right)=\sqrt{3}\left(y+\frac{l_{1} \varepsilon c_{y} t}{2 \sqrt{3}}\right) .
\end{gathered}
$$

Taking $v_{x}=1-\varepsilon \tilde{c}_{x}$ and $v_{y}=\varepsilon \tilde{c}_{y}$, it follows that if $\tilde{c}_{x}$ and $\tilde{c}_{y}$ are defined by $\tilde{c}_{x}=c_{x} l_{1}^{2} / 2$ and $\tilde{c}_{y}=c_{y} l_{1} /(2 \sqrt{3})$, then

$$
\tilde{c}_{x}>\frac{\tilde{c}_{y}^{2}}{2}
$$

which is consistent with equation (5.12).

Comparing the derivation of the KP equation (in terms of $u$ ) with the derivation of the BL equation (in terms of $q$ ) it follows that that $q$ and $u$ are related by

$$
q_{x}=w=-2 \operatorname{sgn}\left(\tilde{\sigma}-\frac{1}{3}\right)\left|\tilde{\sigma}-\frac{1}{3}\right|^{1 / 2} u .
$$

Thus travelling waves for KP and BL equations are directly related, provided that their velocities are related via $\tilde{c}_{x}=c_{x} l_{1}^{2} / 2$ and $\tilde{c}_{y}=c_{y} l_{1} /(2 \sqrt{3})$. An explicit lump formula for $q$ is obtained by integrating equations (5.10) and (5.15) with respect to $x$ to find

$$
q=-32\left(\tilde{\sigma}-\frac{1}{3}\right)^{1 / 2}\left[\frac{\left(x^{\prime}-2 k_{R} y^{\prime}\right)}{4\left(x^{\prime}-2 k_{R} y^{\prime}\right)^{2}+16 k_{I}^{2} y^{\prime 2}+1 / k_{I}^{2}}\right] .
$$

The above equations imply that as $r \rightarrow \infty, u \sim O\left(1 / r^{2}\right), q \sim O(1 / r)$, where $r^{2}=x^{\prime 2}+y^{\prime 2}$.

Next, we will briefly discuss the centre of mass integral relation in the context of the KP and the KdV equations in terms of one of their (less well-known) conserved quantities. Writing equation (2.6) when $H=0$ in a convenient non-dimensional form (or alternatively taking $k_{j} \rightarrow 0 ; j=1,2$ in (4.1)), we find

$$
\frac{\partial}{\partial t} \int_{-\infty}^{\infty} \int_{-\infty}^{\infty} x \eta \mathrm{d} x \mathrm{~d} y=\int_{-\infty}^{\infty} \int_{-\infty}^{\infty} q_{x}(1+\varepsilon \eta) \mathrm{d} x \mathrm{~d} y
$$

Using the coordinates $\xi, T$, as well as conservation of mass $\partial / \partial t \int_{-\infty}^{\infty} \int_{-\infty}^{\infty} \eta \mathrm{d} \xi \mathrm{d} y=0$, the left-hand side of (5.17) takes the form

$$
\frac{\partial}{\partial t} \int_{-\infty}^{\infty} \int_{-\infty}^{\infty} \xi \eta \mathrm{d} \xi \mathrm{d} y=\varepsilon \frac{\partial}{\partial T} \int_{-\infty}^{\infty} \int_{-\infty}^{\infty} \xi \eta \mathrm{d} \xi \mathrm{d} y+\int_{-\infty}^{\infty} \int_{-\infty}^{\infty} \eta \mathrm{d} \xi \mathrm{d} y .
$$

Using (5.2) in the $\xi, T$ variables and dropping $O\left(\varepsilon^{2}\right)$ terms, it follows that

$$
\eta \sim q_{\xi}-\varepsilon q_{T}-\frac{\varepsilon}{2}\left(q_{\xi}\right)^{2}+\tilde{\sigma} \mu^{2} q_{\xi \xi \xi} .
$$

Assuming (as is standard when dealing with KP conservation laws) that $\int_{-\infty}^{\xi} w \mathrm{~d} \xi^{\prime} \rightarrow 0$ rapidly as $|\xi| \rightarrow \infty$, and integrating by parts, we find

$$
\int_{-\infty}^{\infty} \int_{-\infty}^{\infty} \eta \mathrm{d} \xi \mathrm{d} y \sim \int_{-\infty}^{\infty} \int_{-\infty}^{\infty}\left(w+\varepsilon \xi w_{T}-\frac{\varepsilon}{2} w^{2}\right) \mathrm{d} \xi \mathrm{d} y
$$


Then, using the KP equation (5.7), and another integration by parts, we obtain

$$
\int_{-\infty}^{\infty} \int_{-\infty}^{\infty} \eta \mathrm{d} \xi \mathrm{d} y \sim \int_{-\infty}^{\infty} \int_{-\infty}^{\infty}\left(w+\frac{\varepsilon}{2} w^{2}\right) \mathrm{d} \xi \mathrm{d} y .
$$

Therefore, from (5.18) and $\eta \sim q_{\xi}$, the left-hand side of (5.17) becomes

$$
\frac{\partial}{\partial t} \int_{-\infty}^{\infty} x \int_{-\infty}^{\infty} \eta \mathrm{d} x \mathrm{~d} y \sim \varepsilon \frac{\partial}{\partial T} \int_{-\infty}^{\infty} \xi w \mathrm{~d} \xi \mathrm{d} y+\int_{-\infty}^{\infty}\left(w+\frac{\varepsilon}{4} w^{2}\right) \mathrm{d} \xi \mathrm{d} y .
$$

On the other hand, the right-hand side of (5.17) is given by

$$
\int_{-\infty}^{\infty} \int_{-\infty}^{\infty} q_{x}(1+\varepsilon \eta) \mathrm{d} x \mathrm{~d} y \sim \int_{-\infty}^{\infty} \int_{-\infty}^{\infty}\left(w+\varepsilon w^{2}\right) \mathrm{d} \xi \mathrm{d} y .
$$

Therefore, equating the right-hand sides of equations (5.19) and (5.20) we find the integral relation

$$
\frac{\partial}{\partial T} \int_{-\infty}^{\infty} \int_{-\infty}^{\infty} \xi w \mathrm{~d} \xi \mathrm{d} y=\frac{3}{4} \int_{-\infty}^{\infty} \int_{-\infty}^{\infty} w^{2} \mathrm{~d} \xi \mathrm{d} y .
$$

This equation can also be verified directly using the KP equation (5.7). In terms of the KP equation (5.9) in 'standard' variables, the above integral relation can be rewritten (using the change of variables (5.8)) in terms of the following conservation law:

$$
\frac{\partial}{\partial t} \int_{-\infty}^{\infty} \int_{-\infty}^{\infty}\left(x u-3 t u^{2}\right) \mathrm{d} x \mathrm{~d} y=0
$$

Equation (5.22) gives the motion of the centre of mass of the solution $u$ in terms of the constant of motion $\int_{-\infty}^{\infty} u^{2} \mathrm{~d} x$.

If we consider the reduction of the $\mathrm{KP}$ equation to the $\mathrm{KdV}$ equation by taking $\partial / \partial y \rightarrow 0$, then the same analysis applies and the $\mathrm{KdV}$ equation has conservation law identical to (5.21)-(5.22) except that the integration over $y$ is omitted.

Thus, the centre of mass integral relation (5.17) which is derived from the non-local free surface equation, agrees asymptotically with a conservation law obtained directly from the $\mathrm{KP} / \mathrm{KdV}$ equations.

We mention that in Craig \& Groves (1994) the series expansion of the DirichletNeumann map was used to derive small-amplitude/long-wave equations for the Boussinesq and KP equations without surface tension.

\section{The deep water limit}

The goal of this section is to derive from the non-local formulation the smallamplitude, slowly varying envelope equation in deep water for quasi-monochromatic waves. This gives rise to the nonlinear Schrodinger (NLS) equation, which agrees with results obtained directly from the water wave equations (Zakharov 1968).

The $(1+1)$ non-local integral equation $\left(\mathrm{I}^{\prime}\right)$ of $\S 1$ can be written as

$$
\int_{-\infty}^{\infty} \mathrm{e}^{-\mathrm{i} k x}\left\{\mathrm{e}^{-k(\eta+h)}\left(\mathrm{i} \eta_{t}+q_{x}\right)+\mathrm{e}^{k(\eta+h)}\left(\mathrm{i} \eta_{t}-q_{x}\right)\right\} \mathrm{d} x=0,
$$

where we have replaced $k$ by $-k$. 
Taking the limit $h \rightarrow \infty$, and dropping exponentially small terms, we find

$$
k>0, \int_{-\infty}^{\infty} \mathrm{e}^{-\mathrm{i} k x} \mathrm{e}^{k \eta}\left(\mathrm{i} \eta_{t}-q_{x}\right) \mathrm{d} x=0 ; \quad k<0, \int_{-\infty}^{\infty} \mathrm{e}^{-\mathrm{i} k x} \mathrm{e}^{-k \eta}\left(\mathrm{i} \eta_{t}+q_{x}\right) \mathrm{d} x=0 .
$$

For convenience we replace $\eta$ and $q$ by $\varepsilon \eta$ and $\varepsilon q$, and for the small-amplitude limit we take $\varepsilon \ll 1$. The above equation, as well as Bernoulli's equation (4.2) in $1+1$ dimensions, become

$$
\begin{array}{r}
\int_{-\infty}^{\infty} \mathrm{d} x \mathrm{e}^{-\mathrm{i} k x} \mathrm{e}^{\varepsilon|k| \eta}\left[\mathrm{i} \eta_{t}-(\operatorname{sgn}(k)) q_{x}\right]=0, \\
q_{t}+g \eta-\frac{\sigma}{\rho} \frac{\eta_{x x}}{\left(1+\varepsilon^{2} \eta_{x}^{2}\right)^{3 / 2}}+\frac{1}{2} \varepsilon q_{x}^{2}-\frac{\varepsilon}{2} \frac{\left(\eta_{t}+\varepsilon \eta_{x} q_{x}\right)^{2}}{1+\varepsilon^{2} \eta_{x}^{2}}=0 .
\end{array}
$$

Alternatively we can obtain equation (6.1) as a reduction from the two-plus-onedimensional deep water equation (ID) in $\S 2$. Expanding up to $O\left(\varepsilon^{2}\right)$, these equations become

$$
\begin{gathered}
\int_{-\infty}^{\infty} \mathrm{d} x \mathrm{e}^{-\mathrm{i} k x}\left[1+\varepsilon|k| \eta+\varepsilon^{2}|k|^{2} \frac{\eta^{2}}{2}+\cdots\right]\left[\mathrm{i} \eta_{t}-(\operatorname{sgn}(k)) q_{x}\right]=0, \\
q_{t}+g \eta-\frac{\sigma}{\rho}\left(1-\frac{3}{2} \varepsilon^{2} \eta_{x}^{2}\right) \eta_{x x}+\frac{1}{2} \varepsilon q_{x}^{2}-\frac{\varepsilon}{2}\left(\eta_{t}^{2}+2 \varepsilon \eta_{t} \eta_{x} q_{x}\right)+\cdots=0 .
\end{gathered}
$$

Differentiating the second equation with respect to $x$ and letting $q_{x}=Q$, we find

$$
\begin{gathered}
\int_{-\infty}^{\infty} \mathrm{d} x \mathrm{e}^{-i k x}\left[1+\varepsilon|k| \eta+\varepsilon^{2}|k|^{2} \frac{\eta^{2}}{2}+\cdots\right]\left[i \eta_{t}-(\operatorname{sgn}(k)) Q\right]=0, \\
Q_{t}+g \eta_{x}-\frac{\sigma}{\rho} \partial_{x}\left[\eta_{x x}-\frac{3}{2} \varepsilon^{2} \eta_{x}^{2} \eta_{x x}\right]+\varepsilon Q Q_{x}-\frac{\varepsilon}{2} \partial_{x}\left[\eta_{t}^{2}+2 \varepsilon \eta_{t} \eta_{x} Q\right]+\cdots=0 .
\end{gathered}
$$

We now look for solutions in terms of a quasi-monochromatic wave expansion. This takes the form of a Fourier-like series of slowly varying envelopes, multiplied by rapidly varying carrier waves. The first few terms are given by

$$
\begin{gathered}
\eta=\eta_{1} \mathrm{e}^{\mathrm{i} \theta}+\bar{\eta}_{1} \mathrm{e}^{-\mathrm{i} \theta}+\varepsilon\left(\eta_{0}+\eta_{2} \mathrm{e}^{2 \mathrm{i} \theta}+\bar{\eta}_{2} \mathrm{e}^{-2 \mathrm{i} \theta}\right)+\cdots, \\
Q=Q_{1} \mathrm{e}^{\mathrm{i} \theta}+\bar{Q}_{1} \mathrm{e}^{-\mathrm{i} \theta}+\varepsilon\left(Q_{0}+Q_{2} \mathrm{e}^{2 \mathrm{i} \theta}+\bar{Q}_{2} \mathrm{e}^{-2 \mathrm{i} \theta}\right)+\cdots,
\end{gathered}
$$

where

$\eta_{j}=\eta_{j}(X, T), \quad Q_{j}=Q_{j}(X, T), j=0,1,2 \cdots, \quad X=\varepsilon x, \quad T=\varepsilon t, \quad \theta=k_{0} x-w_{0} t$.

We then substitute the above expansions (6.5) and (6.6) into equations (6.3) and (6.4). The analysis of these types of quasi-monochromatic wave expansions in differential equations, such as Bernoulli's equation (6.4), is well understood. However the occurrence of the non-local terms requires some additional mathematical analysis. Substituting equations (6.5) and (6.6) into the non-local equation (6.3) leads to a sequence of integrals of the form:

$$
\int_{-\infty}^{\infty} \mathrm{d} x \mathrm{e}^{-\mathrm{i} k x} F_{m}(X=\varepsilon x) \mathrm{e}^{\mathrm{i} m \theta}, \quad m=0,1,2 \ldots
$$

By the Riemann-Lesbeque lemma, all such integrals are exponentially small except for the term associated with $k=m k_{0}$ (we assume that all functions $\eta_{j}, Q_{j}, j=0,1,2 \ldots$, are rapidly decaying and infinitely smooth). Owing to the arbitrariness of the functions $\eta_{j}, Q_{j}, j=0,1,2 \ldots$, we take $F_{m}=0$. 
Then, equations (6.3) and (6.4) transform to the following equations, in terms of the multiscale variables $t, T=\varepsilon t, x, X=\varepsilon x$ (keeping terms of order $\varepsilon$ ):

$$
\begin{gathered}
\int_{-\infty}^{\infty} \mathrm{d} x \mathrm{e}^{-\mathrm{i} k x}\left[1+\varepsilon|k| \eta+\varepsilon^{2}|k|^{2} \frac{\eta^{2}}{2}+\cdots\right]\left[i\left(\eta_{t}+\varepsilon \eta_{T}\right)-(\operatorname{sgn}(k)) Q\right]=0, \\
Q_{t}+\varepsilon Q_{T}+g\left(\eta_{x}+\varepsilon \eta_{X}\right)-\frac{\sigma}{\rho}\left[\left(1-\frac{3}{2} \varepsilon^{2} \eta_{x}{ }^{2}\right)\left(\partial_{x}+\varepsilon \partial_{X}\right)^{3} \eta-3 \varepsilon^{2} \eta_{x x}^{2} \eta_{x}\right] \\
+\varepsilon Q\left(Q_{x}+\varepsilon Q_{X}\right)-\varepsilon\left[\left(\eta_{t}+\varepsilon \eta_{T}\right)\left(\eta_{x t}+\varepsilon\left(\eta_{t X}+\eta_{x T}\right)+\varepsilon\left(\eta_{t} \eta_{x} Q\right)_{x}\right]+\cdots=0 .\right.
\end{gathered}
$$

To illustrate ideas and fix notation, we derive the leading-order contributions from the above equations:

$$
\begin{gathered}
\int_{-\infty}^{\infty} \mathrm{d} x \mathrm{e}^{-\mathrm{i} k x}\left[\left(\omega_{0} \eta_{1} \mathrm{e}^{\mathrm{i} \theta}+\text { c.c. }\right)-\operatorname{sgn}(k)\left(Q_{1} \mathrm{e}^{\mathrm{i} \theta}+\text { c.c. }\right)+\cdots\right]=0, \\
\left(-\mathrm{i} \omega_{0} Q_{1} \mathrm{e}^{\mathrm{i} \theta}+\text { c.c. }\right)+g\left(\mathrm{i} k_{0} \eta_{1} \mathrm{e}^{\mathrm{i} \theta}+\text { c.c. }\right)-\frac{\sigma}{\rho}\left(\left(\mathrm{i} k_{0}\right)^{3} \eta_{1} \mathrm{e}^{\mathrm{i} \theta}+\text { c.c. }\right)+\cdots=0,
\end{gathered}
$$

where c.c. denotes complex conjugate. From equation (6.10) we find

$$
Q_{1}=\frac{g k_{0}}{\omega_{0}}\left(1+\hat{\sigma} k_{0}^{2}\right) \eta_{1},
$$

where $\hat{\sigma}=\sigma / \rho g$. Letting $k=k_{0}$ in equation (6.9), and using equation (6.11), we obtain

$$
\int_{-\infty}^{\infty} \mathrm{d} x\left\{\eta_{1}\left[\omega-\operatorname{sgn}(k)\left(\frac{g k}{\omega}\right)\left(1+\hat{\sigma} k^{2}\right)\right]\right\}=0,
$$

which in turn implies the dispersion relation for water waves in deep water,

$$
\omega^{2}=g k \operatorname{sgn}(k)\left(1+\hat{\sigma} k^{2}\right) .
$$

Repeating this procedure for the second-harmonic terms $\mathrm{e}^{2 \mathrm{i} \theta}$, we find (from (6.8) and (6.7) respectively)

$$
\begin{array}{r}
2 \omega Q_{2}=2 k g\left(1+4 k^{2} \hat{\sigma}\right) \eta_{2}+\omega^{2} k \eta_{1}{ }^{2}+k Q_{1}{ }^{2}, \\
2 \omega \eta_{2}=\operatorname{sgn}(k) Q_{2}-2 \omega|k| \eta_{1}{ }^{2}+2 k \eta_{1} Q_{1} .
\end{array}
$$

From the mean terms at $\mathrm{e}^{\mathrm{i} 0}$, we find that $Q_{0}=O(\varepsilon), \eta_{0}=O(\varepsilon)$, i.e the mean terms can be neglected to this order of approximation. We can solve for $Q_{2}, \eta_{2}$ from equations (6.13) and (6.14). To the order of approximation we are considering, we can use equation (6.11) to replace $Q_{1}$ by $\eta_{1}$,

$$
\begin{aligned}
Q_{2} & =\frac{1+k^{2} \hat{\sigma}}{1-2 k^{2} \hat{\sigma}} 2 \omega k \eta_{1}{ }^{2}, \\
\eta_{2} & =\frac{1+k^{2} \hat{\sigma}}{1-2 k^{2} \hat{\sigma}}|k| \eta_{1}{ }^{2} .
\end{aligned}
$$

We next calculate the fundamental-harmonic terms $\mathrm{e}^{\mathrm{i} \theta}$. After using equations (6.15) and (6.16), we find from equation (6.8)

$$
Q_{1}=\left(c_{1}+\varepsilon \mathscr{L}_{1}+\varepsilon^{2} \mathscr{L}_{2}\right) \eta_{1}+k^{2} \operatorname{sgn}(k) \frac{1-\frac{1}{2} k^{2} \hat{\sigma}}{1+k^{2} \hat{\sigma}}\left|\eta_{1}\right|^{2} \eta_{1},
$$


where

$$
\begin{gathered}
\mathscr{L}_{1}=\frac{c_{1}}{\mathrm{i} \omega} \partial_{T}+c_{2} \partial_{X}, \\
\mathscr{L}_{2}=\frac{c_{2}}{\mathrm{i} \omega} \partial_{X} \partial_{T}-\frac{c_{1}}{\omega^{2}} \partial_{T}{ }^{2}+c_{3} \partial_{X}{ }^{2}, \\
c_{1}=\frac{g k\left(1+\hat{\sigma} k^{2}\right)}{\omega}, \quad c_{2}=\frac{g\left(1+3 \hat{\sigma} k^{2}\right)}{\mathrm{i} \omega}, \quad c_{3}=-\frac{3 g k \hat{\sigma}}{\omega} .
\end{gathered}
$$

Substituting $Q_{2}, \eta_{2}$, and the above formula for $Q_{1}$, into the non-local equation (6.7), we find that $\eta_{1}$ satisfies the following nonlinear Schrödinger equation:

$$
\mathrm{i}\left(\partial_{T}+v_{g} \partial_{X}\right) \eta_{1}+\varepsilon \frac{\omega^{\prime \prime}(k)}{2} \partial_{X}^{2} \eta_{1}+\varepsilon M\left|\eta_{1}\right|^{2} \eta_{1}=0
$$

where

is the group velocity and

$$
v_{g}=\omega^{\prime}(k)=g \operatorname{sgn}(k) \frac{1+3 k^{2} \hat{\sigma}}{2 \omega},
$$

$$
\begin{gathered}
M=-\frac{\omega k^{2}\left(8+k^{2} \hat{\sigma}+2\left(k^{2} \hat{\sigma}\right)^{2}\right)}{4\left(1+k^{2} \hat{\sigma}\right)\left(1-2 k^{2} \hat{\sigma}\right)}, \\
\omega^{\prime \prime}(k)=-\frac{\omega\left[1-6 k^{2} \hat{\sigma}-3\left(k^{2} \hat{\sigma}\right)^{2}\right]}{4 k^{2}\left(1+k^{2} \hat{\sigma}\right)} .
\end{gathered}
$$

Finally by defining the new variables $\tau=\varepsilon T, \xi=X-v_{g} T$, we find the nonlinear Schrödinger equation in a more standard form, without the small parameter appearing in the equation. This equation, which governs quasi-monochromatic, deep water waves with surface tension (see also Zakharov 1968), is

$$
\mathrm{i} \partial_{\tau} \eta_{1}+\frac{\omega^{\prime \prime}(k)}{2} \partial_{\xi}^{2} \eta_{1}+M\left|\eta_{1}\right|^{2} \eta_{1}=0
$$

A similar analysis can be carried out for quasi-monochromatic waves in finite depth and for three-dimensional water waves. The calculations are much more tedious; that study is outside the scope of this paper.

\section{Computational studies $-2+1$ dimensional lumps}

In this section we develop computational fixed point methods for finding travelling wave solutions for the $2+1$ dimensional water wave equations. These techniques are an extension of methods used in nonlinear optics (cf. Ablowitz \& Musslimani 2003, 2005) and are used to find lump-soliton-type solutions of the KadomtsevPetviashvili, Benney-Luke, and fully nonlinear two plus one dimensional water wave equations. Methods to obtain one-plus-one-dimensional solitary waves are discussed in Appendix B.

We begin by first considering the case of lump-type travelling waves which obey the Benney-Luke (BL) equation with surface tension, i.e. equation (5.5). For convenience we repeat (5.5):

$$
q_{t t}-\tilde{\Delta} q+\mu^{2}\left(\tilde{\sigma}-\frac{1}{3}\right) \tilde{\Delta}^{2} q+\varepsilon\left(|\tilde{\nabla} q|^{2}\right)_{t}+\varepsilon\left(q_{t} \tilde{\Delta} q\right)=0 .
$$

In order to find travelling wave solutions, we assume a travelling wave moving in an arbitrary $x-y$ direction

$$
q(x, y, t)=q\left(x-v_{x} t, y-\gamma^{2} v_{y} t\right)=q\left(x^{\prime}, y^{\prime}\right) .
$$


Substituting this ansatz into the BL equation, we find

$$
\begin{aligned}
v_{x}^{2} q_{x^{\prime} x^{\prime}} & +2 v_{x} v_{y} \gamma^{2} q_{x^{\prime} y^{\prime}}+v_{y}^{2} \gamma^{4} q_{y^{\prime} y^{\prime}}-\left(q_{x^{\prime} x^{\prime}}+\gamma^{2} q_{y^{\prime} y^{\prime}}\right) \\
& +\mu^{2}\left(\tilde{\sigma}-\frac{1}{3}\right)\left(q_{x^{\prime} x^{\prime} x^{\prime} x^{\prime}}+2 \gamma^{2} q_{x^{\prime} x^{\prime} y^{\prime} y^{\prime}}+\gamma^{4} q_{y^{\prime} y^{\prime} y^{\prime} y^{\prime}}\right) \\
= & \varepsilon\left[\left(v_{x} \partial_{x^{\prime}}+\gamma^{2} v_{y} \partial_{y^{\prime}}\right)\left(q_{x^{\prime}}^{2}+\gamma^{2} q_{y^{\prime}}^{2}\right)+\left(\left(v_{x} \partial_{x^{\prime}}+\gamma^{2} v_{y} \partial_{y^{\prime}}\right) q\right)\left(q_{x^{\prime} x^{\prime}}+\gamma^{2} q_{y^{\prime} y^{\prime}}\right)\right] .
\end{aligned}
$$

Solutions to the above equation are obtained using the spectral renormalization method (Ablowitz \& Musslimani 2005). The essence of the method is to transform the underlying equation governing the solitary wave into Fourier space and to find nonlinear non-local integral equation(s), coupled to an algebraic equation(s). The algebraic equation is solved by standard techniques and the integral equation is solved iteratively. The coupling prevents the numerical scheme from diverging.

To develop the iteration scheme, we take the Fourier transform of (7.2), where the Fourier transform of $q$, denoted by $\hat{q}$, is given by

$$
\hat{q}=\iint \mathrm{e}^{-\mathrm{i} k x-\mathrm{i} l y} q(x, y, t) \mathrm{d} x \mathrm{~d} y .
$$

The function $\hat{q}$ satisfies the equation

$$
\begin{aligned}
\hat{q} & =\varepsilon \frac{\mathrm{i}\left(v_{x} k+\gamma^{2} v_{y} l\right)\left(\widehat{q_{x^{\prime}}^{2}}+\gamma^{2} \widehat{q_{y^{\prime}}^{2}}\right)+v_{x}\left(\widehat{q_{x^{\prime}} q_{x^{\prime} x^{\prime}}}+\gamma^{2} \widehat{q_{x^{\prime}} q_{y^{\prime} y^{\prime}}}\right)+\gamma^{2} v_{y}\left(\widehat{q_{y^{\prime}} q_{x^{\prime} x^{\prime}}}+\gamma^{2} \widehat{q_{y^{\prime}} q_{y^{\prime} y^{\prime}}}\right)}{D} \\
& \equiv \mathscr{K}[\hat{q}],
\end{aligned}
$$

where

$$
D=k^{2}\left(1-v_{x}^{2}\right)+\gamma^{2} l^{2}\left(1-\gamma^{2} v_{y}^{2}\right)-2 \gamma^{2} v_{x} v_{y} k l+\left(\tilde{\sigma}-\frac{1}{3}\right) \mu^{2}\left(k^{2}+\gamma^{2} l^{2}\right)^{2} .
$$

We remark that when $\gamma^{2}=\varepsilon$ and $v_{x}=1-\varepsilon \tilde{c}_{x}, v_{y}=\tilde{c}_{y},|\varepsilon| \ll 1$, then we see that $D>0$, provided $\tilde{c}_{x}>\tilde{c}_{y}^{2} / 2$. This inequality agrees with equation (5.14) obtained from the exact lump solution of the KP equation (5.10). By employing the change of variables $q=\alpha p$, we construct the following iteration scheme for $n=0,1,2 \cdots$ :

$$
\hat{p}_{n+1}=\alpha_{n} \mathscr{K}\left[\hat{p}_{n}\right]
$$

with

$$
\alpha_{n}=\frac{\iint\left|\hat{p}_{n}\right|^{2} \mathrm{~d} k \mathrm{~d} l}{\iint \hat{p}_{n}^{*} \mathscr{K}\left[\hat{p}_{n}\right] \mathrm{d} k \mathrm{~d} l} .
$$

The iteration scheme is supplemented by initial functions for $p_{0}$. In practice, the schemes are relatively insensitive to the choice of the initial functions; e.g. hyperbolic secant or Gaussian profiles are adequate in the case of decaying solutions.

A convenient check of these results is to compare the results of the BL (variable $q$ ) equation with the known lump solution results of KP (variable $u$ - see equations (5.9) and (5.10), where $c_{x}, c_{y}$ are the $x-y \mathrm{KP}$ velocities respectively). From $\S 6$, we have the following formulae for the KP lump solution at the origin (recall $\tilde{\sigma}>1 / 3$ ):

$$
\begin{gathered}
u(0,0)=16 k_{I}^{2}=\frac{4}{3}\left(c_{x}-\frac{c_{y}^{2}}{12}\right), \\
q_{x}=Q=-2\left(\tilde{\sigma}-\frac{1}{3}\right)^{1 / 2} u,
\end{gathered}
$$


where the BL velocities $\left(v_{x}, v_{y}\right)$,

$$
v_{x}=1-\varepsilon \tilde{c}_{x}, \gamma^{2} v_{y}=\gamma^{2} \tilde{c}_{y},
$$

are connected to KP velocities $\left(c_{x}, c_{y}\right)$, by

$$
\tilde{c}_{x}=\frac{c_{x}}{2}\left(\tilde{\sigma}-\frac{1}{3}\right)^{1 / 2}, \tilde{c}_{y}=\frac{c_{y}}{2 \sqrt{3}}\left(\tilde{\sigma}-\frac{1}{3}\right)^{1 / 4} .
$$

The same numerical method used to find the travelling wave solution for the BL equation also works for KP. The KP equation also provides a check of the numerical method used herein since it admits an explicit lump solution. Looking for a travelling wave solution of the KP equation (5.9) of the form $u=u\left(x^{\prime}, y^{\prime}\right)$ with $\left(\tilde{\sigma}-\frac{1}{3}\right)>0$ and $x^{\prime}=x-c_{x} \tau, y^{\prime}=y-c_{y} \tau$, we obtain the equation

$$
-c_{x} u_{x^{\prime} x^{\prime}}-c_{y} u_{x^{\prime} y^{\prime}}+u_{x^{\prime} x^{\prime} x^{\prime} x^{\prime}}-3 u_{y^{\prime} y^{\prime}}+3\left(u^{2}\right)_{x^{\prime} x^{\prime}}=0 .
$$

Taking the Fourier transform of equation (7.10), using the standard Fourier notation

$$
\hat{u}=\iint \mathrm{e}^{-\mathrm{i} k x-\mathrm{i} l y} u(x, y, t) \mathrm{d} x \mathrm{~d} y,
$$

we find that $\hat{u}$ satisfies

$$
\hat{u}=\frac{3 k^{2}}{D_{1}} \hat{u^{2}} \equiv \mathscr{G}[\hat{u}]
$$

where

$$
D_{1}=c_{x} k^{2}+c_{y} k l+k^{4}+3 l^{2}=k^{2}\left(c_{x}-\frac{1}{12} c_{y}^{2}\right)+3\left(l+\frac{k c_{y}}{6}\right)^{2}+k^{4} .
$$

Note that $D_{1}>0$ when $\left(c_{x}-\frac{1}{12} c_{y}^{2}\right)>0$, which follows from the known result for KP lumps (see (5.12)).

By employing the change of variables $u=\alpha v$, we construct the following iteration scheme with $n=0,1,2 \ldots$ :

with

$$
\hat{v}_{n+1}=\alpha_{n} \mathscr{G}\left[\hat{v}_{n}\right]
$$

$$
\alpha_{n}=\frac{\iint\left|\hat{v}_{n}\right|^{2} \mathrm{~d} k \mathrm{~d} l}{\iint \hat{v}_{n}^{*} \mathscr{G}\left[\hat{v}_{n}\right] \mathrm{d} k \mathrm{~d} l} .
$$

In figure 1 we present a typical example of a wave profile associated with the KP and BL equations; plotted are the $y=0, x=0$ profiles respectively, when $c_{x}=3, c_{y}=0$. The exact solution is obtained from equation (5.10), and the numerical results are found using the method introduced in this section. The numerical solution associated with KP cannot be distinguished from the theoretical solution in the graph; the numerical scheme correctly reproduces the known KP lump-soliton solution. We have plotted the wave profiles at $y=0, x=0$ resp. corresponding to the associated 'KP' function

$$
u=-\frac{q_{x}}{2\left(\tilde{\sigma}-\frac{1}{3}\right)^{1 / 2}} .
$$

The figure shows that KP is a good approximation to $\mathrm{BL}$ in this range of parameters.

Figure 2 shows a nearly linear relationship between $u$ and $c_{x}$ (note in figure 2, $v_{x}=1-\varepsilon \tilde{c}_{x}, v_{y}=0$; see (7.9) for the relationship between $c_{x}$ and $\tilde{c}_{x}$ ). Comparison with $\mathrm{KP}$ theory is given by the solid line. Even at $\mu=0.5$, the KP solution provides a good 


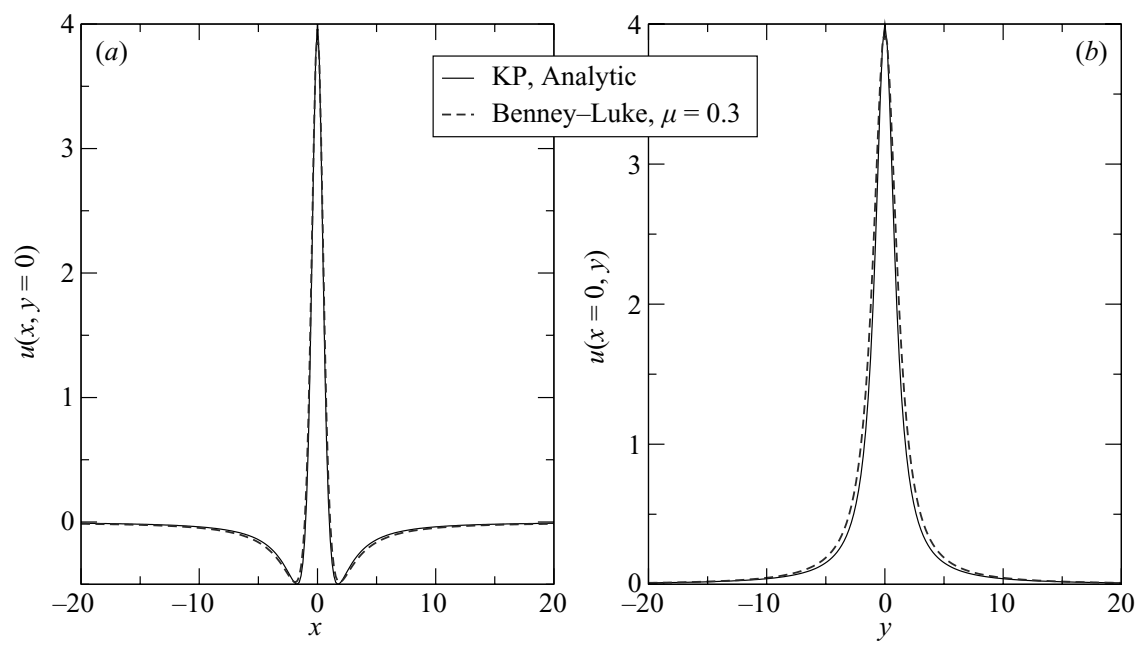

Figure 1. Wave profile at $(a) y=0$ and $(b) x=0$ for the BL (5.5) and KP equations (5.9) with $\tilde{\sigma}=2 / 3, c_{x}=3, c_{y}=0$.

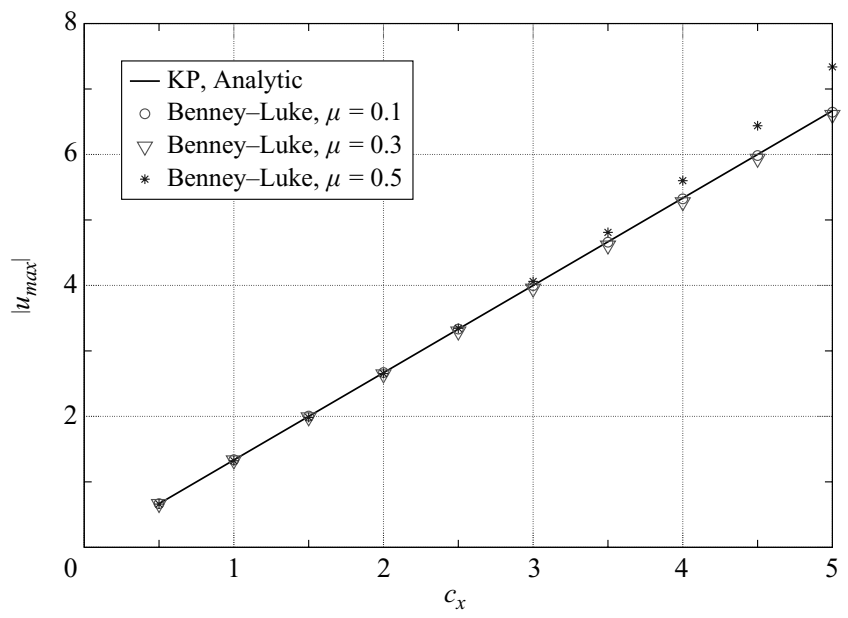

FIGURE 2. $u(0,0)=u_{\max }$ vs. $c_{x}$ for various values of $\mu$. This figure shows that the KP theory is a good approximation to the BL equation in this range of parameters.

approximation to the BL lump solution though we see that there is some deviation at large values of $c_{x}$.

Next, we look for two-dimensional travelling wave lumps associated with the full two-plus-one-dimensional water wave equations. First, for convenience of standard notation, we slightly modify equation (4.4) by redefining: $k_{1}=-k, k_{2}=-l, x_{1}=x$, $x_{2}=y$ and $\kappa=\sqrt{k^{2}+\gamma^{2} l^{2}}$. This yields

$$
\begin{aligned}
\mathrm{i} \cosh [\kappa \mu] \hat{\eta}_{t}-\sinh [\kappa \mu]\left[\left(\frac{k}{\kappa \mu}\right)\left(\widehat{q_{x}}\right)+\left(\frac{\gamma^{2} l}{\kappa \mu}\right)\left(\widehat{q_{y}}\right)\right] \\
=\iint \mathrm{d} x \mathrm{~d} y \mathrm{e}^{-\mathrm{i}(k x+l y)}\left\{\mathrm{i} \eta_{t}(\cosh [\kappa \mu]-\cosh [\kappa \mu(1+\varepsilon \eta)])\right. \\
\left.-(\sinh [\kappa \mu]-\sinh [\kappa \mu(1+\varepsilon \eta)])\left[\frac{k}{\kappa \mu} q_{x}+\frac{\gamma^{2} l}{\kappa \mu} q_{y}\right]\right\},
\end{aligned}
$$


where we now define the Fourier transforms of $\eta$ as

$$
\hat{\eta}=\iint \mathrm{e}^{-\mathrm{i} k x-\mathrm{i} l y} \eta(x, y, t) \mathrm{d} x \mathrm{~d} y,
$$

and similarly for the Fourier transform of the derivatives of $q$. We treat (4.5) similarly and go to travelling wave coordinates, $x^{\prime}-v_{x} t, y^{\prime}=y-v_{y} t$ and then we drop the primes:

$$
\begin{aligned}
& -\cosh (\kappa \mu)\left(v_{x} k+v_{y} l\right) \hat{\eta}+\frac{\sinh (\kappa \mu) \mathrm{i} \kappa}{\mu} \hat{q} \\
& =\iint \mathrm{d} x \mathrm{~d} y \mathrm{e}^{-\mathrm{i}(k x+l y)}(-\mathrm{i})\left(v_{x} \partial_{x}+v_{y} \partial_{y}\right) \eta[\cosh [\kappa \mu(1+\varepsilon \eta)]-\cosh (\kappa \mu)] \\
& \quad+\iint \mathrm{d} x \mathrm{~d} y \mathrm{e}^{-\mathrm{i}(k x+l y)}\left[\frac{\sinh (\kappa \mu)-\sinh [\kappa \mu(1+\varepsilon \eta)]}{\kappa \mu}\right]\left[k q_{x}+\gamma^{2} l q_{y}\right]=R_{1 f}, \quad \text { (7.16) } \\
& \quad-\left(v_{x} \partial_{x}+v_{y} \partial_{y}\right) q+\eta=-\frac{\varepsilon}{2}\left(q_{x}^{2}+\gamma^{2} q_{y}^{2}\right)+\frac{\varepsilon \mu^{2}}{2 \delta}\left[-\left(v_{x} \partial_{x}+v_{y} \partial_{y}\right) \eta+\varepsilon q_{x} \eta_{x}+\varepsilon \gamma^{2} q_{y} \eta_{y}\right]^{2} \\
& \quad+\frac{\tilde{\sigma}}{\delta^{3 / 2}}\left[\mu^{2} \eta_{x x}\left(1+(\varepsilon \mu \gamma)^{2} \eta_{y}^{2}\right)+(\mu \gamma)^{2} \eta_{y y}\left(1+(\varepsilon \mu)^{2} \eta_{x}^{2}\right)-2\left(\varepsilon \mu^{2} \gamma\right)^{2} \eta_{x} \eta_{y} \eta_{x y}\right], \quad \text { (7.17) }
\end{aligned}
$$

where

$$
\delta=1+(\varepsilon \mu)^{2}\left(\eta_{x}^{2}+\gamma^{2} \eta_{y}^{2}\right) .
$$

We rewrite the above equation by putting all linear dispersive terms on the left-hand side and taking the Fourier transform; as with the BL equation we assume $q$ has a well-defined Fourier transform. This yields

$$
\begin{aligned}
& -\mathrm{i}\left(v_{x} k+v_{y} l\right) \hat{q}+\left(1+\tilde{\sigma} \mu^{2} \kappa^{2}\right) \hat{\eta} \\
& =\iint \mathrm{d} x \mathrm{~d} y \mathrm{e}^{-\mathrm{i}(k x+l y)}\left\{-\frac{\varepsilon}{2}\left(q_{x}^{2}+\gamma^{2} q_{y}^{2}\right)+\frac{\varepsilon \mu^{2}}{2 \delta}\left[-\left(v_{x} \partial_{x}+v_{y} \partial_{y}\right) \eta+\varepsilon q_{x} \eta_{x}+\varepsilon \gamma^{2} q_{y} \eta_{y}\right]^{2}\right. \\
& \quad \times \frac{\tilde{\sigma}}{\delta^{3 / 2}}\left[\mu^{2} \eta_{x x}\left(1+(\varepsilon \mu \gamma)^{2} \eta_{y}^{2}\right)+(\mu \gamma)^{2} \eta_{y y}\left(1+(\varepsilon \mu)^{2} \eta_{x}^{2}\right)-2\left(\varepsilon \mu^{2} \gamma\right)^{2} \eta_{x} \eta_{y} \eta_{x y}\right. \\
& \left.\left.\quad-\delta^{3 / 2}\left(\mu^{2} \eta_{x x}+(\mu \gamma)^{2} \eta_{y y}\right)\right]\right\}=R_{2 f} .
\end{aligned}
$$

Hence $\hat{\eta}, \hat{q}$ are given by

$$
\begin{gathered}
\hat{\eta}=\frac{\left(v_{x} k+v_{y} l\right) R_{1 f}+\frac{\sinh (\kappa \mu) \kappa}{\mu} R_{2 f}}{J_{w w}}=R_{3 f}, \\
\hat{q}=\frac{\left(1+\tilde{\sigma} \mu^{2} \kappa^{2}\right) R_{1 f}+\cosh (\kappa \mu)\left(v_{x} k+v_{y} l\right) R_{2 f}}{\mathrm{i} J_{w w}}=R_{4 f},
\end{gathered}
$$

where

$$
J_{w w}=\left(1+\tilde{\sigma} \mu^{2} \kappa^{2}\right) \frac{\sinh (\kappa \mu) \kappa}{\mu}-\cosh (\kappa \mu)\left(v_{x} k+v_{y} l\right)^{2} .
$$

The spectral renormalization numerical technique proceeds by making use of the following change of variables:

$$
\eta=\alpha \psi, \quad q=\beta P .
$$

This yields

$$
\hat{\psi}=\frac{R_{3 f}}{\alpha}, \quad \hat{P}=\frac{R_{4 f}}{\beta} .
$$


In order to find equations for $\alpha$ and $\beta$ we multiply the above equations by $\hat{\psi}^{*}$ and $\hat{P}^{*}$ respectively, and integrate in $k$. This yields the following algebraic system satisfied by $\alpha$ and $\beta$ :

where

$$
F(\alpha, \beta)=\|\hat{P}\|^{2}, \quad G(\alpha, \beta)=\|\hat{\psi}\|^{2},
$$

$$
\begin{aligned}
& F(\alpha, \beta)=\frac{1}{\beta} \iint \hat{P}^{*} R_{4 f}[\beta \hat{P}, \alpha \hat{\psi}] \mathrm{d} k \mathrm{~d} l, \\
& G(\alpha, \beta)=\frac{1}{\alpha} \iint \hat{\psi}^{*} R_{3 f}[\beta \hat{P}, \alpha \hat{\psi}] \mathrm{d} k \mathrm{~d} l .
\end{aligned}
$$

The iteration scheme then takes the form

with

$$
\widehat{\psi_{n+1}}=\frac{R_{3 f}\left[\beta_{n} \hat{P}_{n}, \alpha_{n} \hat{\psi}_{n}\right]}{\alpha_{n}}, \quad \widehat{P_{n+1}}=\frac{R_{4 f}\left[\beta_{n} \hat{P}_{n}, \alpha_{n} \hat{\psi}_{n}\right]}{\beta_{n}},
$$

$$
F\left(\alpha_{n}, \beta_{n}\right)=\left\|\hat{P}_{n}\right\|^{2}, \quad G\left(\alpha_{n}, \beta_{n}\right)=\left\|\hat{\psi}_{n}\right\|^{2},
$$

where $n=0,1,2 \ldots$, and at each iteration step we solve for $\alpha_{n}, \beta_{n}$ from the above system of equations for $F, G$. The iteration scheme is supplemented by initial functions for $P_{0}, \psi_{0}$. As mentioned earlier, the schemes are relatively insensitive to the choice of the initial functions; e.g. hyperbolic secant or Gaussian profiles are adequate in the case of decaying solutions. In order to establish a connection with the BL equation, we assume that $v_{x}=1-\varepsilon \tilde{c}_{x}$ and $v_{y}=\gamma^{2} \tilde{c}_{y}$. When $\varepsilon=\gamma^{2}=\mu^{2} \ll 1$, the condition (5.10), $c_{x}>\frac{1}{12} c_{y}^{2}$, ensures that $J_{w w}>0$.

We also note that higher-order nonlinear approximations to full water waves can be obtained. Such approximations are useful when we find fixed-point solutions to the full water wave equations. A quadratic nonlinear approximation is obtained from the full water wave equations by expanding the hyperbolic functions $\sinh [\kappa \mu(1+$ $\varepsilon \eta)], \cosh [\kappa \mu(1+\varepsilon \eta)]$ for small $\varepsilon, \mu$, and keeping only quadratic nonlinear terms. We find from the above equations the following quadratic and quintic approximations.

Quadratic:

$$
\begin{gathered}
-\cosh [\kappa \mu]\left(v_{x} k+v_{y} l\right) \hat{\eta}+\frac{\mathrm{i} \kappa}{\mu} \sinh [\kappa \mu] \hat{q}=\iint \mathrm{d} x \mathrm{~d} y \mathrm{e}^{-\mathrm{i}(k x+l y)}\left\{-\mathrm{i}\left(v_{x} \eta_{x}+v_{y} \eta_{y}\right)\right. \\
\left.\times \sinh [\kappa \mu] \kappa \mu \varepsilon \eta-\kappa \mu \varepsilon \eta \cosh [\kappa \mu]\left[\frac{k}{\kappa \mu} q_{x}+\frac{\gamma^{2} l}{\kappa \mu} q_{y}\right]\right\}=R_{1 Q} \\
-\mathrm{i}\left(v_{x} k+v_{y} l\right) \hat{q}+\left(1+\tilde{\sigma} \mu^{2} \kappa^{2}\right) \hat{\eta} \\
=\iint \mathrm{d} x \mathrm{~d} y \mathrm{e}^{-\mathrm{i}(k x+l y)}\left[-\frac{\varepsilon}{2}\left(q_{x}^{2}+\gamma^{2} q_{y}^{2}\right)+\frac{\varepsilon \mu^{2}}{2}\left(v_{x} \eta_{x}+v_{y} \eta_{y}\right)^{2}\right]=R_{2 Q}
\end{gathered}
$$

Quintic:

$$
\begin{aligned}
- & \cosh [\kappa \mu]\left(v_{x} k+v_{y} l\right) \hat{\eta}+\frac{\mathrm{i} \kappa}{\mu} \sinh [\kappa \mu] \hat{q} \\
= & \iint \mathrm{d} x \mathrm{~d} y \mathrm{e}^{-\mathrm{i}(k x+l y)}\left\{-\mathrm{i}\left(v_{x} \eta_{x}+v_{y} \eta_{y}\right)\left[\sinh [\kappa \mu] \kappa \mu \varepsilon \eta+\cosh [\kappa \mu] \frac{(\kappa \mu \varepsilon \eta)^{2}}{2}\right.\right. \\
& \left.+\sinh [\kappa \mu] \frac{(\kappa \mu \varepsilon \eta)^{3}}{6}\right]-\left[\frac{k}{\kappa \mu} q_{x}+\frac{\gamma^{2} l}{\kappa \mu} q_{y}\right]\left[\cosh [\kappa \mu] \kappa \mu \varepsilon \eta+\sinh [\kappa \mu] \frac{(\kappa \mu \varepsilon \eta)^{2}}{2}\right. \\
& \left.\left.+\cosh [\kappa \mu] \frac{(\kappa \mu \varepsilon \eta)^{3}}{6}\right]\right\} \\
= & R_{1 Q Q},
\end{aligned}
$$



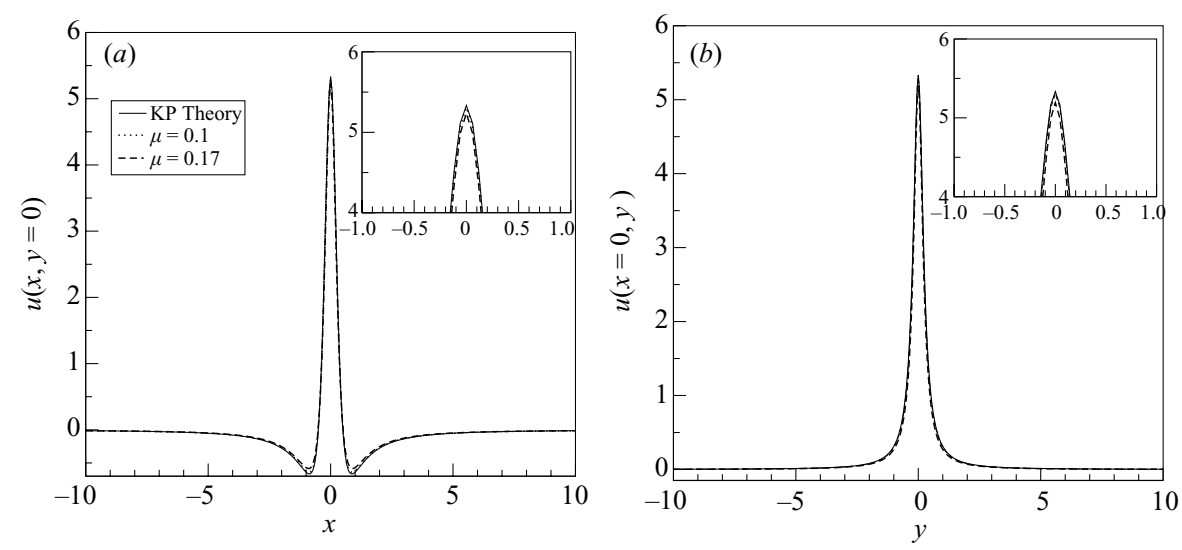

FIGURE 3. Wave profile at $(a) y=0$ and $(b) x=0$ for the full water wave equations (7.16) and (7.18) with $\tilde{\sigma}=2 / 3$. The function $u$ is plotted using (7.14) and the velocities are $v_{x}=1-\varepsilon \tilde{c}_{x}, v_{y}=0$ with $c_{x}=4.0$ using the relationship (7.9). This figure demonstrates that Benney-Luke/KP equations are good approximations to the full water wave equations. The inset shows an enlarged view of the peak of the wave, highlighting the difference in maximum amplitudes.

$$
\begin{aligned}
& -\mathrm{i}\left(v_{x} k+v_{y} l\right) \hat{q}+\left(1+\tilde{\sigma} \mu^{2} \kappa^{2}\right) \hat{\eta} \\
& =\iint \mathrm{d} x \mathrm{~d} y \mathrm{e}^{-\mathrm{i}(k x+l y)}\left\{-\frac{\varepsilon}{2}\left(q_{x}^{2}+\gamma^{2} q_{y}^{2}\right)\right. \\
& \quad+\frac{\varepsilon \mu^{2}}{2}\left[-\left(v_{x} \eta_{x}+v_{y} \eta_{y}\right)+\varepsilon\left(q_{x} \eta_{x}+\gamma^{2} q_{y} \eta_{y}\right)\right]^{2}-\frac{\varepsilon\left(\varepsilon \mu^{2}\right)^{2}}{2}\left(\eta_{x}^{2}+\gamma^{2} \eta_{y}^{2}\right)\left(v_{x} \eta_{x}+v_{y} \eta_{y}\right)^{2} \\
& \left.\quad+\tilde{\sigma}\left[\left(\varepsilon \mu^{2} \gamma\right)^{2}\left(\eta_{x x} \eta_{y}^{2}+\eta_{y y} \eta_{x}^{2}-2 \eta_{x} \eta_{y} \eta_{x y}\right)-\frac{3}{2}\left(\varepsilon \mu^{2}\right)^{2}\left(\eta_{x}^{2}+\gamma^{2} \eta_{y}^{2}\right)\left(\eta_{x x}+\gamma^{2} \eta_{y y}\right)\right]\right\} \\
& =R_{2 Q Q} .
\end{aligned}
$$

The procedure to find a fixed point of the quadratic-quintic systems is similar with that for full water waves with $R_{f}$ replaced by $R_{Q}, R_{Q Q}$, respectively; hence we do not explicitly give it in detail.

In figures 3 and 4 solutions of the full water wave equations are depicted. Numerical solutions are obtained employing the method described earlier; theoretical values are calculated from (5.10). In figure 3 , the wave profiles at $y=0, x=0$ respectively, corresponding to the associated 'KP' function $u$ obtained from (7.14), are also given. The dotted lines lie on top of the theoretical result hence are difficult to discern. The solutions of the quadratic and quintic approximations are similar to those found for the full water waves, hence they are not given here. It is outside the scope of this paper to find lump solitary waves of the highest amplitude.

\section{Conclusions}

In this paper, a coupled system of non-local nonlinear equations on a fixed domain is derived which governs the classical free boundary equations of water waves in $2+1$ dimensions with constant depth. The extension of this system to the case of varying depth is also obtained. These compact equations are explicit and in spectral form which is useful in calculations as described in $\S \S 2,5-7$. In addition, integral relations 


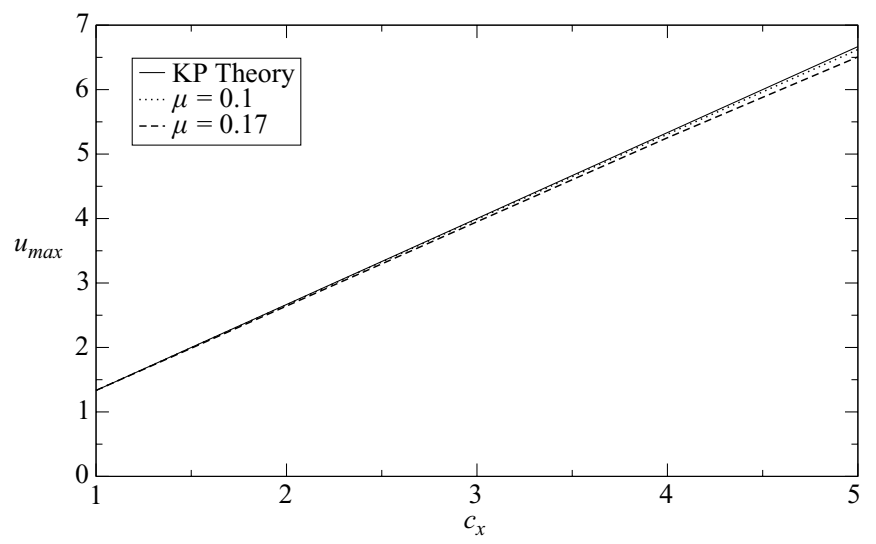

FIGURE 4. $u(0,0)=u_{\max }$ vs. $c_{x}$ the full water wave equations (7.16) and (7.18) for various values of $\mu$ using the same parameters as figure 3. This figure also shows that the Benney-Luke/KP equations are good approximations to full water waves for $\mu=0.1$.

are deduced which include conservation of mass and centre of mass and virial-like formulae.

These equations are shown to admit shallow water/long wave, and deep water asymptotic equations, which include the Boussinesq and Benney-Luke equations for long waves and the nonlinear Schrodinger equation in deep water.

A numerical technique is introduced for computing solitary-lump solutions for the Benney-Luke and the non-local $2+1$-dimensional water wave equations. The Fourier nature of the equations is useful in the computational evaluation of lump solitary waves.

This work was partially supported by NSF grant DMS 0303756, and by the EPSRC. We thank Douglas Baldwin for pointing out the conservation law (A 11) and Andrew Docherty for computational support.

\section{Appendix A. The ' $1+1$ ' long-wave reduction}

In this Appendix we consider the long-wave reduction where all variables are assumed to be independent of $x_{2}$. The $1+1$-dimensional limits are obtained from the Boussinesq and the KP equations by letting $\gamma=0$ in the long-wave equations derived in $\S 5$. We will subsequently use these equations to compare the numerical results obtained from the full $1+1$-dimensional water wave equations.

Equations (5.1) and (5.2) imply that the 1+1-dimensional Boussinesq system is given by the equations

$$
\begin{gathered}
\left(1-\frac{\mu^{2}}{2} \partial_{x x}\right) \eta_{t}+\left(\partial_{x x}-\frac{\mu^{2}}{6} \partial_{x x x x}\right) q+\varepsilon\left(\eta_{x} q_{x}+\eta q_{x x}\right)=0, \\
\eta=-q_{t}-\frac{\varepsilon}{2}\left(q_{x}\right)^{2}+\tilde{\sigma} \mu^{2} \eta_{x x} .
\end{gathered}
$$

It is worth noting that the above system is equivalent to the classical equations of Boussinesq. Indeed, defining $Q=q_{x}$, the above equations yield

$$
\left(1-\frac{\mu^{2}}{2} \partial_{x x}\right) \eta_{t}+\left(\partial_{x}-\frac{\mu^{2}}{6} \partial_{x x x}\right) Q+\varepsilon(Q \eta)_{x}=0,
$$




$$
\eta_{x}=-Q_{t}-\frac{\varepsilon}{2}\left(Q^{2}\right)_{x}+\tilde{\sigma} \mu^{2} \eta_{x x x}
$$

Letting $Q=\tilde{Q}-\mu^{2} \frac{1}{3} \tilde{Q}_{x x}+\ldots$, equations (A1)-(A2) yield (to this order of approximation),

$$
\begin{gathered}
\eta_{t}+\tilde{Q}_{x}+\varepsilon(\tilde{Q} \eta)_{x}=0 \\
\tilde{Q}_{t}+\eta_{x}+\varepsilon \tilde{Q} \tilde{Q}_{x}-\mu^{2} \tilde{\sigma} \eta_{x x x}-\frac{\mu^{2}}{3} \tilde{Q}_{x x t}=0 .
\end{gathered}
$$

We use $\tilde{Q}_{t} \sim-\eta_{x}$ and $\eta_{x x} \sim \eta_{t t}$, which results in the following modification of equation (A4):

$$
\tilde{Q}_{t}+\eta_{x}+\varepsilon \tilde{Q} \tilde{Q}_{x}-\mu^{2}\left(\tilde{\sigma}-\frac{1}{3}\right) \eta_{x t t}=0 .
$$

Equations (A3) and (A5) are the classical Boussinesq equations in normalized form, with surface tension included. We also note that these equations yield the one dimensional form of the Benney-Luke equation (5.5), with the addition of surface tension,

$$
q_{t t}-q_{x x}+\mu^{2}\left(\tilde{\sigma}-\frac{1}{3}\right) q_{x x x x}+\varepsilon\left(\partial_{t} q_{x}^{2}+q_{t} q_{x x}\right)=0 .
$$

From either the Boussinesq equations (A3)-(A.5), or equation (A6), we can derive the Korteweg-deVries (KdV) equation. In (A6) we take $\mu^{2}=\varepsilon, \xi=x-t, \quad T=\varepsilon t$ and $q=q(\xi, T ; \varepsilon)$. Then letting $w=Q=q_{\xi}$, we find

$$
2 w_{T}+\left(\frac{1}{3}-\tilde{\sigma}\right) w_{\xi \xi \xi}+3 w w_{\xi}=0 .
$$

Equation (A7) has a solitary wave solution of the form

$$
w=Q=q_{\xi}=A \operatorname{sech}^{2}\left[\beta\left(\xi-c_{1} T\right)\right], \quad c_{1}=\frac{A}{2}=2\left(\frac{1}{3}-\tilde{\sigma}\right) \beta^{2} .
$$

Note also that $\xi-c_{1} T=x-c t$, where $c=1+\varepsilon c_{1}$.

The change of coordinates given by equation (5.8) yields the $\mathrm{KdV}$ equation in standard form

$$
u_{\tau}+u_{x x x}+6 u u_{x}=0
$$

for which the soliton solution is given by

$$
u=2 \kappa^{2} \operatorname{sech}^{2}\left[\kappa\left(x-4 \kappa^{2} \tau-x_{0}\right)\right] .
$$

Finally, we remind the reader of the conservation relationship (5.21) that also follows asymptotically from the $1+1$-dimensional reduction of equation (2.6),

$$
\frac{\partial}{\partial T} \int_{-\infty}^{\infty} \xi w \mathrm{~d} \xi=\frac{3}{4} \int_{-\infty}^{\infty} w^{2} \mathrm{~d} \xi
$$

Equation (A10) can also be verified by using the KdV equation (A7). In terms of the KdV equation (A9) in 'standard' variables, the above integral relation can be rewritten (using the change of variables (5.8)) in terms of the following conservation law:

$$
\frac{\partial}{\partial t} \int_{-\infty}^{\infty}\left(x u-3 t u^{2}\right) \mathrm{d} x=0
$$

Equation (A11) gives the motion of the centre of mass of the solution $u$ in terms of the constant of motion $\int_{-\infty}^{\infty} u^{2} \mathrm{~d} x$. Thus, the centre of mass integral relation (A10)-(A11) which is derived from the non-local free surface equation agrees asymptotically with a conservation law obtained from the $\mathrm{KdV}$ equation. 


\section{Appendix B. Solitary waves in 1+1 dimensions - computational studies}

In this Appendix we discuss computational fixed-point methods for finding travelling wave solutions for the $1+1$-dimensional water wave equations. They are employed to find travelling wave solutions of Boussinesq type, and the fully nonlinear $1+1$-dimensional water wave equations associated with equation $\left(\mathrm{I}^{\prime}\right)$ given in the introduction. The Boussinesq system is a limiting case of the water wave equations, and the solitary wave results obtained from the full $1+1$ system properly reduce to the Boussinesq solitary waves.

The $1+1$ non-local equation governing Laplace's equation (1.1) and the boundary conditions (1.2)-(1.3) is equation $\left(\mathrm{I}^{\prime}\right)$. If we replace $x_{1}$ by $x$ and take the $1+1$ reduction in Bernoulli's equation (1.8), we find the following equations:

$$
\begin{gathered}
\int_{-\infty}^{\infty} \mathrm{d} x \mathrm{e}^{\mathrm{i} k x}\left\{\mathrm{i} \eta_{t} \cosh k[\eta+h]+q_{x} \sinh k[\eta+h]\right\}=0, \\
q_{t}+\frac{1}{2} q_{x}^{2}-\frac{1}{2} \frac{\left(\eta_{t}+\eta_{x} q_{x}\right)^{2}}{1+\eta_{x}^{2}}+g \eta=\frac{\sigma}{\rho} \frac{\eta_{x x}}{\left(1+\eta_{x}^{2}\right)^{3 / 2}} .
\end{gathered}
$$

If we introduce non-dimensional quantities, as in $\S 5$, and if for convenience we replace $k$ with $-k$, we find

$$
\begin{gathered}
\int_{-\infty}^{\infty} \mathrm{d} x \mathrm{e}^{-\mathrm{i} k x}\left\{\mathrm{i} \eta_{t} \cosh [k \mu(1+\varepsilon \eta)]-\frac{q_{x}}{\mu} \sinh [k \mu(1+\varepsilon \eta)]\right\}=0 \\
q_{t}+\eta+\frac{\varepsilon}{2} q_{x}^{2}=\frac{\varepsilon \mu^{2}}{2} \frac{\left(\eta_{t}+\varepsilon q_{x} \eta_{x}\right)^{2}}{1+\varepsilon^{2} \mu^{2} \eta_{x}^{2}}+\frac{\tilde{\sigma} \mu^{2} \eta_{x x}}{\left[1+\varepsilon^{2} \mu^{2} \eta_{x}^{2}\right]^{3 / 2}}
\end{gathered}
$$

where $\tilde{\sigma}=\sigma / \rho g h^{2}$.

We modify (B 1) and (B 2) by adding/subtracting the linear terms, taking the derivative and Fourier transform of (B 2) and taking $Q=q_{x}$. This yields

$$
\begin{aligned}
& \mathrm{i} \hat{\eta}_{t} \cosh [k \mu]+\hat{Q} \frac{\sinh [k \mu]}{\mu}= \int_{-\infty}^{\infty} \mathrm{d} x \mathrm{e}^{-\mathrm{i} k x}\left\{\mathrm{i} \eta_{t}(\cosh [k \mu]-\cosh [k \mu(1+\varepsilon \eta)])\right. \\
&\left.-\frac{Q}{\mu}(\sinh [k \mu(1+\varepsilon \eta)]-\sinh [k \mu])\right\}=0 \\
& \hat{Q}_{t}+\mathrm{i} k \hat{\eta}=\mathrm{i} k \int_{-\infty}^{\infty} \mathrm{d} x \mathrm{e}^{-\mathrm{i} k x}\left[-\frac{\varepsilon Q^{2}}{2}+\frac{\varepsilon \mu^{2}}{2} \frac{\left(\eta_{t}+\varepsilon Q \eta_{x}\right)^{2}}{1+\varepsilon^{2} \mu^{2} \eta_{x}^{2}}+\frac{\tilde{\sigma} \mu^{2} \eta_{x x}}{\left(1+\varepsilon^{2} \mu^{2} \eta_{x}^{2}\right)^{3 / 2}}\right] .
\end{aligned}
$$

Letting $\xi=x-c t$, thus $\eta_{t}=-c \eta_{\xi}, q_{x}=q_{\xi}=Q, q_{t}=-c Q$, yields

$$
\begin{array}{r}
-c \cosh (\mu k) \hat{\eta}(k)+\frac{\sinh (\mu k)}{\mu k} \hat{Q}(k)=\frac{\mathrm{i} c}{k} \int_{-\infty}^{\infty} \mathrm{d} \xi \mathrm{e}^{-\mathrm{i} k \xi}\{\cosh (\mu k)-\cosh [\mu k(1+\varepsilon \eta)]\} \eta_{\xi} \\
+\frac{1}{\mu k} \int_{-\infty}^{\infty} \mathrm{d} \xi \mathrm{e}^{-\mathrm{i} k \xi}\{\sinh (\mu k)-\sinh [\mu k(1+\varepsilon \eta)]\} Q(\xi), \quad \text { (B 3) } \\
-c \hat{Q}(k)+\left(1+\tilde{\sigma} \mu^{2} k^{2}\right) \hat{\eta}(k)=-\frac{1}{2} \int_{-\infty}^{\infty} \mathrm{d} \xi \mathrm{e}^{-\mathrm{i} k \xi}\left[\frac{\varepsilon Q^{2}-\varepsilon \mu^{2} c^{2} \eta_{\xi}^{2}+2 c(\varepsilon \mu)^{2} Q \eta_{\xi}^{2}}{1+(\varepsilon \mu)^{2} \eta_{\xi}^{2}}\right] \\
+\tilde{\sigma} \mu^{2} \int_{-\infty}^{\infty} \mathrm{d} \xi \frac{\mathrm{e}^{-\mathrm{i} k \xi} \eta_{\xi \xi}\left(1-\left(1+(\varepsilon \mu)^{2} \eta_{\xi}^{2}\right)^{3 / 2}\right)}{\left(1+(\varepsilon \mu)^{2} \eta_{\xi}^{2}\right)^{3 / 2}},
\end{array}
$$


where hats denote the Fourier transform, i.e.

$$
\hat{\eta}=\int_{-\infty}^{\infty} \mathrm{e}^{-\mathrm{i} k x} \eta(x, t) \mathrm{d} x,
$$

and similarly for $\hat{Q}$.

First, let us consider the limiting cases for which $\varepsilon=\mu^{2}$, and $\varepsilon \rightarrow 0$. By expanding the $\cosh [\mu k(1+\varepsilon \eta)]$ and $\sinh [\mu k(1+\varepsilon \eta)]$ functions in powers of $\varepsilon$, we find

$$
\begin{aligned}
\cosh [\mu k(1+\varepsilon \eta)]=\cosh (\mu k)+\varepsilon \mu k \eta \sinh (\mu k) & +\frac{(\varepsilon \mu k \eta)^{2}}{2} \cosh (\mu k) \\
& +\frac{(\varepsilon \mu k \eta)^{3}}{6} \sinh (\mu k)+\cdots, \\
\sinh [\mu k(1+\varepsilon \eta)]=\sinh (\mu k)+\varepsilon \mu k \eta \cosh (\mu k) & +\frac{(\varepsilon \mu k \eta)^{2}}{2} \sinh (\mu k) \\
& +\frac{(\varepsilon \mu k \eta)^{3}}{6} \cosh (\mu k)+\cdots
\end{aligned}
$$

Substituting (B 5) and (B 6) into (B 3) and (B 4), we can develop successively higher nonlinear approximations to the water wave equations.

\section{B.1. Quadratic nonlinear approximation-Boussinesq type}

In this section we keep only quadratic nonlinear terms:

$$
\begin{aligned}
& -c \hat{\eta}(k)+b(k) \hat{Q}(k)=-\varepsilon \widehat{\eta Q}+\frac{k c \varepsilon \mu \tanh (\mu k)}{2} \widehat{\eta^{2}}, \\
& -c \hat{Q}(k)+\left(1+\mu^{2} k^{2} \tilde{\sigma}\right) \hat{\eta}(k)=-\frac{\varepsilon}{2} \widehat{Q^{2}}+\frac{c^{2} \varepsilon \mu^{2}}{2} \widehat{\eta_{\xi}^{2}},
\end{aligned}
$$

where $b(k)=\tanh (\mu k) /(\mu k)$.

These equations are also consistent with the above Boussinesq system when $\mu, \varepsilon$ are asymptotically small. In order to find a family of solutions to (B 7) and (B 8), we employ the spectral renormalization iteration scheme.

First, we solve for $\hat{\eta}$ and $\hat{Q}$, to find

$$
\begin{gathered}
\hat{Q}(k)=\frac{1}{J}\left(\varepsilon\left(1+\mu^{2} k^{2} \tilde{\sigma}\right) \widehat{\eta Q}+\frac{c \varepsilon \widehat{Q^{2}}}{2}-\frac{\left(1+\mu^{2} k^{2} \tilde{\sigma}\right) c k \varepsilon \mu \tanh (\mu k) \widehat{\eta^{2}}}{2}-\frac{c^{3} \varepsilon \mu^{2} \widehat{\eta_{\xi}^{2}}}{2}\right), \\
\hat{\eta}(k)=\frac{1}{J}\left(c \varepsilon \widehat{\eta Q}+\frac{b(k) \varepsilon \widehat{Q^{2}}}{2}-\frac{c^{2} k \varepsilon \mu \tanh (\mu k) \widehat{\eta^{2}}}{2}-\frac{c^{2} b(k) \varepsilon \mu^{2} \widehat{\eta_{\xi}^{2}}}{2}\right),
\end{gathered}
$$

where

$$
J=J(k)=c^{2}-\left(1+\mu^{2} k^{2} \tilde{\sigma}\right) b(k) .
$$

Following the same procedure as in $\S 7$ we make the following change of variables:

$$
\hat{\eta}(k)=\alpha \hat{\psi}(k), \quad \hat{Q}(k)=\beta \hat{P}(k),
$$

where $\alpha$ and $\beta$ are constants independent of $k$ to be determined by an algebraic system found below. 
Substituting (B 11) into (B 9) and (B 10), we find

$$
\begin{aligned}
& \hat{P}(k)=\frac{1}{J}\left(\varepsilon\left(1+\mu^{2} k^{2} \tilde{\sigma}\right) \alpha \widehat{\psi P}\right. \\
& \left.+\frac{c \varepsilon \beta \widehat{P^{2}}}{2}-\frac{\left(1+\mu^{2} k^{2} \tilde{\sigma}\right) c k \varepsilon \mu \tanh (\mu k)}{2} \frac{\alpha^{2} \widehat{\psi^{2}}}{\beta}-\frac{c^{3} \varepsilon \mu^{2}}{2} \frac{\alpha^{2} \widehat{\psi_{\xi}^{2}}}{\beta}\right), \\
& \hat{\psi}(k)=\frac{1}{J}\left(c \varepsilon \beta \widehat{\psi P}+\frac{b(k) \varepsilon}{2} \frac{\beta^{2} \widehat{P^{2}}}{\alpha}-\frac{c^{2} k \varepsilon \mu \tanh (\mu k) \alpha \widehat{\psi^{2}}}{2}-\frac{c^{2} b(k) \varepsilon \mu^{2} \alpha \widehat{\psi_{\xi}^{2}}}{2}\right) .
\end{aligned}
$$

Next, multiplying (B 12) by $\hat{P}^{*}$ and (B 13) by $\hat{\psi}^{*}$, and integrating with respect to $k$ we find a coupled algebraic system satisfied by $\alpha$ and $\beta$. For clarity we give this system explicitly:

$$
\begin{aligned}
& a_{1} \alpha+a_{2} \beta-\left(a_{3}+a_{4}\right) \frac{\alpha^{2}}{\beta}=\|\hat{P}\|^{2}, \\
& b_{1} \beta+b_{2} \frac{\beta^{2}}{\alpha}-\left(b_{3}+b_{4}\right) \alpha=\|\hat{\psi}\|^{2},
\end{aligned}
$$

where the $L^{2}$ norm is given by $\|\hat{P}\|^{2}=\int \mathrm{d} k|\hat{P}|^{2}$ and

$$
\begin{gathered}
a_{1}=\int \mathrm{d} k \hat{P}^{*} \varepsilon\left(1+\mu^{2} k^{2} \tilde{\sigma}\right) \frac{\widehat{\psi P}}{J}, \quad a_{2}=\int \mathrm{d} k \hat{P}^{*} \frac{c \varepsilon \widehat{P^{2}}}{2 J}, \\
a_{3}=\int \mathrm{d} k \hat{P}^{*} \frac{c k \varepsilon \mu \tanh (\mu k)\left(1+\mu^{2} k^{2} \tilde{\sigma}\right) \widehat{\psi^{2}}}{2 J}, \quad a_{4}=\int \mathrm{d} k \hat{P}^{*} \frac{c^{3} \varepsilon \mu^{2} \widehat{\psi_{\xi}^{2}}}{2 J}, \\
b_{1}=\int \mathrm{d} k \hat{\phi}^{*} c \varepsilon \frac{\widehat{\psi P}}{J}, \quad b_{2}=\int \mathrm{d} k \hat{\psi}^{*} \frac{b(k) \varepsilon \widehat{P^{2}}}{2 J}, \\
b_{3}=\int \mathrm{d} k \hat{\psi}^{*} \frac{c^{2} k \varepsilon \mu \tanh (\mu k) \widehat{\psi^{2}}}{2 J}, \quad b_{4}=\int \mathrm{d} k \hat{\psi}^{*} \frac{c^{2} b(k) \varepsilon \mu^{2} \widehat{\psi_{\xi}^{2}}}{2 J} .
\end{gathered}
$$

We then iterate (B 12), (B 13), (B 14), (B 15), as follows:

$$
\begin{aligned}
& \hat{P}_{n+1}(k)=\frac{1}{J}\left(\varepsilon\left(1+\tilde{\sigma} \mu^{2} k^{2}\right) \alpha_{n} \widehat{\psi_{n} P_{n}}\right. \\
& \left.+\frac{c \varepsilon \beta_{n} \widehat{P_{n}^{2}}}{2}-\frac{c k \varepsilon \mu \tanh (\mu k)\left(1+\tilde{\sigma} \mu^{2} k^{2}\right)}{2} \frac{\alpha_{n}^{2} \widehat{\psi_{n}^{2}}}{\beta_{n}}-\frac{c^{3} \varepsilon \mu^{2}}{2} \frac{\alpha_{n}^{2} \widehat{\psi_{n \xi}^{2}}}{\beta_{n}}\right), \\
& \hat{\psi}_{n+1}(k)=\frac{1}{J}\left(c \varepsilon \beta_{n} \widehat{\psi_{n} P_{n}}+\frac{b(k) \varepsilon}{2} \frac{\beta_{n}^{2} \widehat{P_{n}^{2}}}{\alpha_{n}}-\frac{c^{2} k \varepsilon \mu \tanh (\mu k) \alpha_{n} \widehat{\psi_{n}^{2}}}{2}-\frac{c^{2} b(k) \varepsilon \mu^{2} \alpha_{n} \widehat{\psi_{n \xi}^{2}}}{2}\right), \\
& a_{1 n} \alpha_{n}+a_{2 n} \beta_{n}-\left(a_{3 n}+a_{4 n}\right) \frac{\alpha_{n}^{2}}{\beta_{n}}=\left\|\hat{P}_{n}\right\|^{2}, \\
& b_{1 n} \beta_{n}+b_{2 n} \frac{\beta_{n}^{2}}{\alpha_{n}}-\left(b_{3 n}+b_{4 n}\right) \alpha_{n}=\left\|\hat{\psi}_{n}\right\|^{2},
\end{aligned}
$$




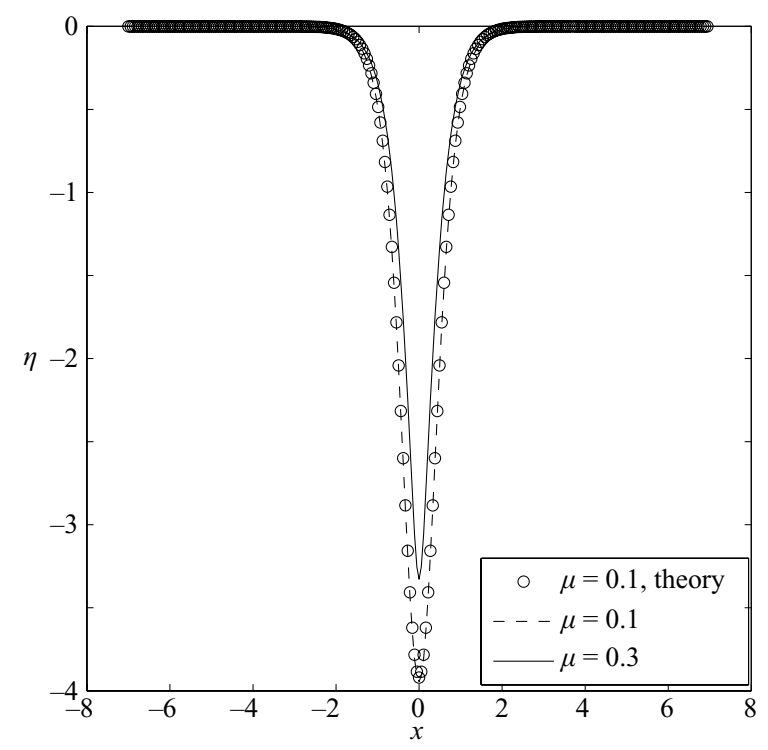

FIGURE 5. Surface amplitude profile for $\tilde{\sigma}=2 / 3$ and various values of $\mu, c_{1}=2$, obtained from (B 7) and (B 8); theoretical values are from (A 8).

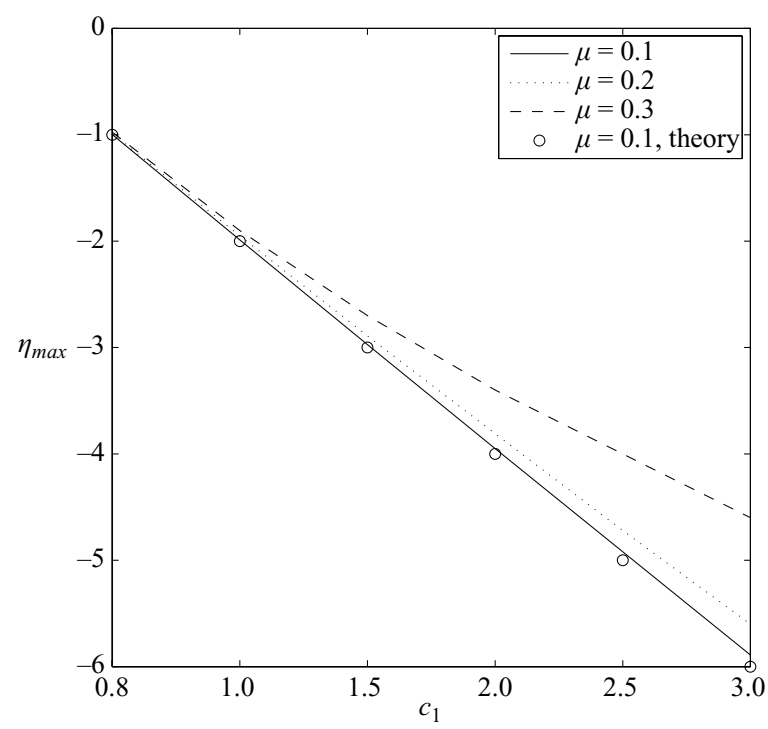

FIGURE 6. Maximum amplitude vs. $c_{1}$ for various values of $\mu, \tilde{\sigma}=2 / 3$, obtained from (B 7) and (B 8); theoretical values are from (A 8).

for $n=0,1,2 \ldots$ The iteration scheme is supplemented by initial functions for $P_{0}, Q_{0}$. As mentioned before, the schemes are relatively insensitive to the choice of the initial functions; e.g. hyperbolic secant or Gaussian profiles are usually adequate in the case of decaying solutions. We compare the results with those obtained by the asymptotic analysis presented earlier - see (A 8).

In figure 5 we show typical examples of the amplitude profile $\eta=\eta(x)$ for several values of the small parameter $\mu$ for a given wave speed $c_{1}=2$ and surface tension $\tilde{\sigma}=2 / 3$ respectively. Figure 6 shows the maximum amplitude of $\eta$ vs. $c_{1}$ for various 
values of the small parameter $\mu$ (note: here we take $c=1-\varepsilon c_{1}$ and $\mu^{2}=\varepsilon$ ) and surface tension $\tilde{\sigma}=2 / 3$ respectively.

B.2. One-dimensional travelling wave solutions obtained from the full water wave equations

In this section we obtain numerical solutions from the full one-plus-one-dimensional non-local water wave equations and compare with the Boussinesq-type equations. We apply the same ideas outlined before, i.e. we substitute (B 11) into (B 3) and (B 4), to find

$$
\begin{gathered}
\hat{P}(k)=\frac{\left(1+\tilde{\sigma} \mu^{2} k^{2}\right) \mu k \mathscr{R}_{1}[\beta P, \alpha \psi]+c \mu k \cosh (\mu k) \mathscr{R}_{2}[\beta P, \alpha \psi]}{\beta\left\{\left(1+\tilde{\sigma} \mu^{2} k^{2}\right) \sinh (\mu k)-c^{2} \mu k \cosh (\mu k)\right\}}, \\
\hat{\psi}(k)=\frac{c \mu k \mathscr{R}_{1}[\beta P, \alpha \psi]+\sinh (\mu k) \mathscr{R}_{2}[\beta P, \alpha \psi]}{\alpha\left\{\left(1+\tilde{\sigma} \mu^{2} k^{2}\right) \sinh (\mu k)-\mu k c^{2} \cosh (\mu k)\right\}},
\end{gathered}
$$

where

$$
\begin{array}{r}
\mathscr{R}_{1}=\frac{\mathrm{i} c}{k} \int_{-\infty}^{\infty} \mathrm{d} \xi \mathrm{e}^{-\mathrm{i} k \xi}\{\cosh (\mu k)-\cosh [\mu k(1+\varepsilon \eta)]\} \eta_{\xi} \\
+\frac{1}{\mu k} \int_{-\infty}^{\infty} \mathrm{d} \xi \mathrm{e}^{-\mathrm{i} k \xi}\{\sinh (\mu k)-\sinh [\mu k(1+\varepsilon \eta)]\} Q(\xi), \\
\mathscr{R}_{2}=-\frac{1}{2} \int_{-\infty}^{\infty} \mathrm{d} \xi \mathrm{e}^{-\mathrm{i} k \xi}\left[\frac{\varepsilon Q^{2}-\varepsilon \mu^{2} c^{2} \eta_{\xi}^{2}+2 c(\varepsilon \mu)^{2} Q \eta_{\xi}^{2}}{1+(\varepsilon \mu)^{2} \eta_{\xi}^{2}}\right] \\
+\tilde{\sigma} \mu^{2} \int_{-\infty}^{\infty} \mathrm{d} \xi \frac{\left.\mathrm{e}^{-\mathrm{i} k \xi} \eta_{\xi \xi}\left[1-(\varepsilon \mu)^{2} \eta_{\xi}^{2}\right)^{3 / 2}\right]}{\left(1+(\varepsilon \mu)^{2} \eta_{\xi}^{2}\right)^{3 / 2}} .
\end{array}
$$

The iteration scheme is given by

$$
\begin{gathered}
\hat{P}_{n+1}(k)=\frac{\left(1+\tilde{\sigma} \mu^{2} k^{2}\right) \mu k \mathscr{R}_{1}\left[\beta_{n} P_{n}, \alpha_{n} \psi_{n}\right]+c \mu k \cosh (\mu k) \mathscr{R}_{2}\left[\beta_{n} P_{n}, \alpha_{n} \psi_{n}\right]}{\beta_{n}\left\{\left(1+\tilde{\sigma} \mu^{2} k^{2}\right) \sinh (\mu k)-c^{2} \mu k \cosh (\mu k)\right\}}, \\
\hat{\psi}_{n+1}(k)=\frac{c \mu k \mathscr{R}_{1}\left[\beta_{n} P_{n}, \alpha_{n} \psi_{n}\right]+\sinh (\mu k) \mathscr{R}_{2}\left[\beta_{n} P_{n}, \alpha_{n} \psi_{n}\right]}{\alpha_{n}\left\{\left(1+\tilde{\sigma} \mu^{2} k^{2}\right) \sinh (\mu k)-\mu k c^{2} \cosh (\mu k)\right\}},
\end{gathered}
$$

where $\alpha_{n}$ and $\beta_{n}$ satisfy the nonlinear system

$$
\begin{gathered}
\int \frac{\left(1+\tilde{\sigma} \mu^{2} k^{2}\right) \mu k \mathscr{R}_{1}\left[\beta_{n} P_{n}, \alpha_{n} \psi_{n}\right]+c \mu k \cosh (\mu k) \mathscr{R}_{2}\left[\beta_{n} P_{n}, \alpha_{n} \psi_{n}\right]}{\beta_{n}\left\{\left(1+\tilde{\sigma} \mu^{2} k^{2}\right) \sinh (\mu k)-c^{2} \mu k \cosh (\mu k)\right\}} \hat{P}_{n}^{*}(k) \mathrm{d} k \\
=\int\left|\hat{P}_{n}\right|^{2}(k) \mathrm{d} k, \quad \\
\int \frac{c \mu k \mathscr{R}_{1}\left[\beta_{n} P_{n}, \alpha_{n} \psi_{n}\right]+\sinh (\mu k) \mathscr{R}_{2}\left[\beta_{n} P_{n}, \alpha_{n} \psi_{n}\right]}{\alpha_{n}\left\{\left(1+\tilde{\sigma} \mu^{2} k^{2}\right) \sinh (\mu k)-\mu k c^{2} \cosh (\mu k)\right\}} \hat{\psi}_{n}^{*}(k) \mathrm{d} k=\int\left|\hat{\psi}_{n}\right|^{2}(k) \mathrm{d} k,
\end{gathered}
$$

for $n=0,1,2 \ldots$ In figure 7 we show typical examples of the amplitude profile $\eta=\eta(x)$ for several values of the parameter $\mu$ for a given wave speed $c_{1}=2$ and surface tension $\tilde{\sigma}=2 / 3$, respectively obtained from (B 3) and (B 4). Figure 8 shows the maximum amplitude of $\eta$ vs. $c_{1}$ for various values of the small parameter $\mu$ (as in $\S$ B. 1 we take $c=1-\varepsilon c_{1}$ and $\mu^{2}=\varepsilon$ ) and surface tension $\tilde{\sigma}=2 / 3$ respectively. The comparisons between the curves depicting maximum amplitude of $\eta$ vs. $c_{1}$, show 


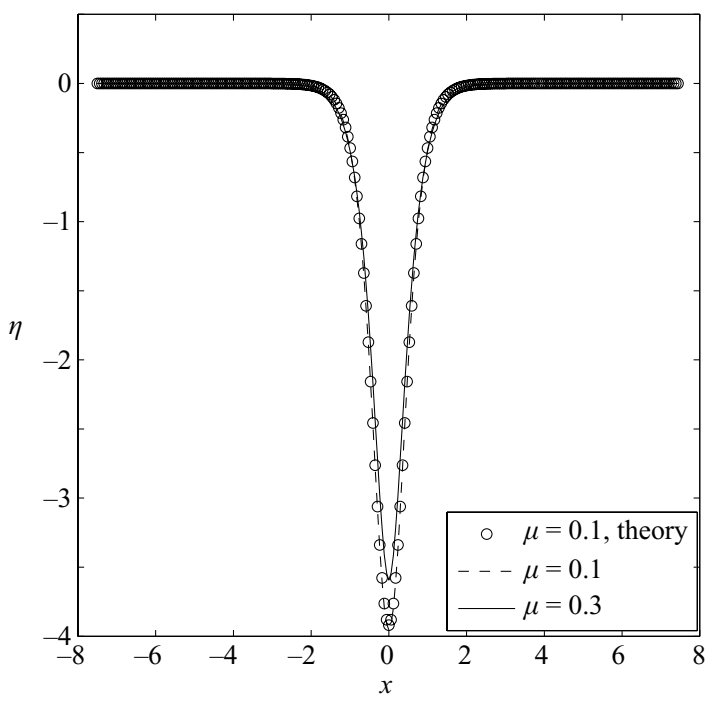

FIGURE 7. Surface amplitude profile for $\tilde{\sigma}=2 / 3$ and various values of $\mu, c_{1}=2$, obtained from (B 3) and (B 4); theoretical values are obtained from (A 8).

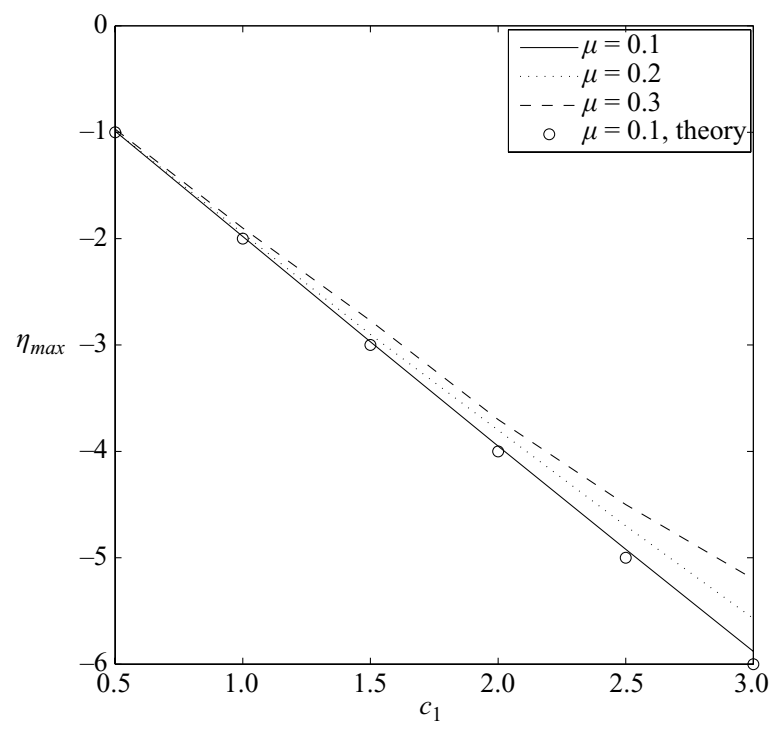

Figure 8. Maximum amplitude vs. $c_{1}$ for $\tilde{\sigma}=2 / 3$ and various values of $\mu$, obtained from (B 3) and (B 4); theoretical values are obtained from (A 8).

distinct differences between the quadratically nonlinear case in figure 6 and the full water wave case in figure 8 .

\section{REFERENCES}

Ablowitz, M. J. \& Clarkson, P. A. 1991 Solitons, Nonlinear Evolution Equations and Inverse Scattering. Cambridge University Press.

Ablowitz, M. J. \& Musslimani, Z. 2003 Discrete spatial solitons in a diffraction managed nonlinear waveguide array: a unified approach. Physica D 184, 276. 
Ablowitz, M. J. \& Musslimani, Z. 2005 A spectral renormalization method to compute selflocalized solutions to nonlinear systems. Optics Lett. 30, 2140-2142.

Bateman, W. J. D., Swan, C. \& Taylor, P. 2001 On the efficient numerical simulation of directionally spread surface water waves. J. Comput. Phys. 174, 277.

Benjamin, T. B. \& Mahony J. J. 1971 On an invariant property of water waves. J. Fluid Mech. 49, 385.

Benjamin, T. B. \& Olver, P. J. 1982 Hamiltonian structure, symmetries and conservation laws for water waves. J. Fluid Mech. 125, 137.

Benney, D. J. \& LuKe, J. C. 1964 Interactions of permanent waves of finite amplitude. J. Maths and Phys. 43, 455.

Berger, K. M. \& Milewski, P. A. 2000 The generation and evolution of lump solitary waves in surface-tension-dominated flows. SIAM J. Appl. Maths 61, 731.

BoussinesQ, M. J. 1877 Essai sur la theorie des eaux courantes. Memoirs Presentees par Diverse Savants a l'Academie des Sciences Inst. France 23, 1-680.

Craig, W. \& Groves, M. D. 1994 Hamiltonian long-wave approximations to the water-wave problem. Wave Motion 19, 367.

Craig, W., Groves, M. D., Schneider, G. \& Toland, J. F. 2002 Recent developments in the mathematical theory of water waves - introduction. Phil. Trans. R. Soc. Lond. A 360, 2107.

Craig, W., Guyenne, P., Nicholls, D. \& Sulem, C. 2005 Hamiltonian long-wave expansions for water waves over a rough bottom. Proc. R. Soc. Lond A 461, 839.

Craig, W. \& Nicholls, D. P. 2000 Travelling two and three dimensional caplillary gravity water waves. SIAM J. Maths Anal. 32, 323.

Craig, W. \& Sulem, C. 1993 Numerical simulation of gravity waves. J. Comput. Phys. 108, 73.

DolD, J. W. 1992 An efficient surface-integral algorithm applied to unsteady gravity waves. J. Comput. Phys. 103, 90.

Falcon, E., Laroache, C. \& Fauve, S. 2002 Observation of depression solitary waves on a thin fluid layer. Phys. Rev. Lett. 89, 1204501.

FoKAS, A. S. 2000 On the integrability of linear and nonlinear partial differential equations. J. Math. Phys. 41, 4188.

FornberG, B. 1980 A numerical method for conformal mapping. SIAM J. Sci. Statist. Comput. 1, 386.

Kadomtsev, B. B. \& Petviashvili, V. I. 1970 On the stability of solitary waves in weakly dispersive media. Sovi. Phys. Dokl. 15, 539.

KorteweG, D. J. \& DE VRIes, G. 1895 On the change of form of long waves advancing in a rectangular canal, and on a new type of long stationary wave. Phil. Mag. 39, 422.

Longuet-Higgins, M. S. \& Cokelet, E. D. 1976 The deformation of steep surface waves on water I. A numerical method of computation. Proc. R. Soc. Lond. A 350, 1.

ZAKHARov, V. E. 1968 Stability of periodic waves of finite amplitude on the surface of a deep fluid. J. Appl. Mech. Tech. Phys. 2, 190.

Zakharov, V. E., Dyachenko, A. I. \& Vasilyev, O. A. 2002 New method for numerical simulation of a nonstationary potential flow of incompressible fluid with a free surface. Eur. J. Mech. B-Fluids 21, 283. 